\title{
The XMM-Newton EPIC background: Production of background maps and event files ${ }^{\star}$
}

\author{
A. M. Read ${ }^{1,2}$ and T. J. Ponman ${ }^{1}$ \\ 1 School of Physics and Astronomy, University of Birmingham, Edgbaston, Birmingham B15 2TT, UK \\ 2 Deptartment of Physics and Astronomy, Leicester University, Leicester LE1 7RH, UK \\ Received 9 April 2003 / Accepted 11 July 2003

\begin{abstract}
XMM-Newton background maps for the 3 EPIC instruments in their different instrument/mode/filter combinations and in several energy bands have been constructed using a superposition of 72 pointed observations. Event datasets, with point sources excised, for the different instrument/mode/filter combinations have also been assembled, with longer exposure times than previously available files. The construction of the background maps and event files, together with their properties and usage are described here. Also given are statistical properties of the photon and particle components of the XMM-Newton EPIC background, based on the analysis of the 72 datasets.
\end{abstract}

Key words. surveys - X-rays: diffuse background - X-rays: general

\section{Introduction}

The XMM-Newton observatory (Jansen et al. 2001) provides unrivalled capabilities for detecting low surface brightness emission features from extended and diffuse galactic and extragalactic sources, by virtue of the large field of view of the X-ray telescopes with the EPIC MOS (Turner et al. 2001) and pn (Strüder et al. 2001) cameras at the foci, and the high throughput yielded by the heavily nested telescope mirrors. The satellite has the largest collecting area of any imaging X-ray telescope.

In order to exploit the excellent EPIC data from extended objects, the EPIC background, known now to be higher than estimated pre-launch, needs to be understood thoroughly. With a good model of the particle and photon background, one can correctly background-subtract images and spectra extracted over different energy bands and from different areas of the detectors.

Here we provide details of a project to use a large number of XMM-Newton pointed observations to help define the EPIC background and to produce background maps for each of the three EPIC instruments (pn, MOS1 \& MOS2) in several different energy bands. Also, significantly improved background event files, useful for spectral analysis, with longer exposures than previously produced files, and specific to several particular instrument/mode/filter combinations, have been created as

Send offprint requests to: A. M. Read,

e-mail: amr30@star.le.ac.uk

* Based on observations with XMM-Newton, an ESA Science Mission with instruments and contributions directly funded by ESA Member States and the USA (NASA). part of the analysis. The co-addition of many fields allows the minimization of any "cosmic variance", resulting from variations in the local diffuse X-ray emission or contamination from pathologically bright sources.

This paper is intended as an aid when studying or working with the EPIC background, and in particular, when using these files. The current understanding of the XMM-Newton background is described briefly in Sect. 2. Section 3 describes the methods used in the creation of the background products. Information on how to use the background images for XMM EPIC background analysis is given in Sect. 4, along with caveats as to their use. The relevant information for the event files can be found in Sect. 5, followed by some concluding remarks.

All the background product files (maps, event files, related software etc.), together with other scripts and procedures for XMM-Newton background analysis are available from http://www.sr.bham.ac.uk/xmm3/

\section{The XMM-Newton EPIC X-ray background}

The EPIC background has been shown (via the work of Lumb et al. 2002 and many others; see Appendix) to comprise solar soft protons, cosmic rays, electronic noise and cosmic X-ray photon background. We now briefly discuss the properties of these contributions:

- Solar soft protons (see Sect. 3.2) perhaps accelerated by "magnetospheric reconnection" events and trapped by the Earth's magnetosphere, then gathered by XMM-Newton's grazing mirrors. These dominate the times of high background. 
Table 1. Summary of the components within the XMM-Newton EPIC Background; temporal, spatial and spectral properties.

\begin{tabular}{|c|c|c|c|c|c|}
\hline & \multicolumn{2}{|c|}{ PARTICLES } & & \multicolumn{2}{|c|}{ PHOTONS } \\
\hline & SOFT PROTONS & $\begin{array}{l}\text { INTERNAL } \\
\text { (Cosmic-ray induced) }\end{array}$ & $\begin{array}{l}\text { ELECTRONIC } \\
\text { NOISE }\end{array}$ & HARD X-RAYS & SOFT X-RAYS \\
\hline Source & $\begin{array}{l}\text { Few } 100 \mathrm{keV} \\
\text { solar protons }\end{array}$ & $\begin{array}{l}\text { Interaction of High } \\
\text { Energy particles } \\
\text { with detector } \\
\end{array}$ & $\begin{array}{l}\text { 1) Bright pixels } \\
\text { 2) Elec. overshoot } \\
\text { near pn readout }\end{array}$ & $\begin{array}{l}\text { X-ray } \\
\text { background } \\
\text { (AGN etc) } \\
\end{array}$ & $\begin{array}{l}\text { Local Bubble } \\
\text { Galactic Disk } \\
\text { Galactic Halo } \\
\end{array}$ \\
\hline $\begin{array}{c}\text { Variable? } \\
\text { (per Obs) } \\
\text { (Obs to } \\
\quad \text { Obs) }\end{array}$ & $\begin{array}{l}\text { Flares }(>1000 \%) \\
\text { Unpredictable. } \\
\text { More far from } \\
\text { apogee. } \\
\text { Low-E flares turn } \\
\text { on before high-E }\end{array}$ & $\begin{array}{l} \pm 10 \% \\
\pm 10 \% \\
\text { No increase after } \\
\text { solar flares }\end{array}$ & $\begin{array}{l} \pm 10 \% \\
1)>1000 \% \text { (pixels } \\
\text { come and go, also } \\
\text { meteor damage) }\end{array}$ & $\begin{array}{l}\text { Constant } \\
\text { Constant }\end{array}$ & $\begin{array}{l}\text { Constant } \\
\text { Variation with } \\
\text { RA/Dec }( \pm 35 \%)\end{array}$ \\
\hline $\begin{array}{l}\text { Spatial } \\
\text { Vignetted? } \\
\text { Structure? }\end{array}$ & $\begin{array}{l}\text { Yes (scattered) } \\
\text { Perhaps, } \\
\text { unpredictable }\end{array}$ & $\begin{array}{l}\text { No } \\
\text { Yes. Detector }+ \\
\text { construction } \\
\text { MOS: outer CCDs more } \\
\text { Al, CCD edges more } \mathrm{Si} \\
\text { PN: Central hole in } \\
\text { high-E lines }(\sim 8 \mathrm{keV})\end{array}$ & $\begin{array}{l}\text { No } \\
\text { Yes } \\
\text { 1) Individual pixels } \\
\text { \& columns } \\
\text { 2) Near pn } \\
\text { readout (CAMEX) }\end{array}$ & $\begin{array}{l}\text { Yes } \\
\text { No }\end{array}$ & $\begin{array}{l}\text { Yes } \\
\text { No, apart from } \\
\text { real astronomical } \\
\text { objects }\end{array}$ \\
\hline Spectral & $\begin{array}{l}\text { Variable } \\
\text { Unpredictable } \\
\text { No correlation } \\
\text { between intensity } \\
+ \text { shape } \\
\text { Low-E flares turn } \\
\text { on before high-E }\end{array}$ & $\begin{array}{l}\text { Flat }+ \text { fluorescence }+ \\
\text { detector noise } \\
\text { MOS: } 1.5 \mathrm{keV} \text { Al-K } \\
1.7 \mathrm{keV} \mathrm{Si-K} \\
\text { det.noise }<0.5 \mathrm{keV} \text {. } \\
\text { High-E - low-intensity } \\
\text { lines }(\mathrm{Cr}, \mathrm{Mn}, \mathrm{Fe}-\mathrm{K}, \mathrm{Au}) \\
\mathrm{PN}: 1.5 \mathrm{keV} \text { Al-K } \\
\text { no } \mathrm{Si}(\text { self-absorbed) } \\
\mathrm{Cu}-\mathrm{Ni}-\mathrm{Zn}-\mathrm{K}(\sim 8 \mathrm{keV}) \\
\text { det.noise }<0.3 \mathrm{keV}\end{array}$ & $\begin{array}{l}\text { 1) low-E }(<300 \mathrm{eV}) \text {, } \\
\text { tail may reach } \\
\text { higher-E } \\
\text { 2) low-E }(<300 \mathrm{eV})\end{array}$ & $\begin{array}{l}\sim 1.4 \text { power law. } \\
\text { Below } 5 \mathrm{keV} \text {, } \\
\text { dominates over } \\
\text { internal } \\
\text { component }\end{array}$ & $\begin{array}{l}\text { Thermal with } \\
\lesssim 1 \mathrm{keV} \text { emission } \\
\text { lines }\end{array}$ \\
\hline
\end{tabular}

During quiescent periods (i.e. with no significant soft proton contamination), the remaining components are:

- High energy, non-vignetted cosmic ray induced events, unrejected by the the on-board electronics. Also, associated instrumental fluorescence, due to the interaction of highenergy particles with the detector.

- Non-vignetted electronic noise: bad (bright) pixels, and dark current, though the latter is thought negligible. In actuality, most of the bright pixels are rejected on board the satellite, and the vast majority of the remaining bad pixels are removed by analysis software on the ground. The amount of "flickering" activity of these remaining pixels is thought to be related to the radiation level of the observation.

- Low to medium energy, vignetted X-ray photons from the sky. These can be divided into the local (predominately soft) X-ray background, the cosmic (harder) X-ray background, and single reflections entering the telecope from bright sources outside of the nominal field of view (FOV). Lumb et al. (2002) estimate that the contribution of diffuse flux gathered from out-of-field angles of 0.4-1.4 degrees is of order $7 \%$ of the true, focussed in-field signal, and the associated systematic error (due mainly to the energy dependence) is $\pm 2 \%$.

Table 1 gives a brief summary of the temporal, spatial and spectral properties of these, the major components contributing to the XMM-Newton EPIC background.

The present analysis is related in several respects to the work of Lumb et al. (2002), and the reader is encouraged to consult this work. Additional notes regarding other related work on the EPIC background can be found in the Appendix.

\section{Data analysis}

Source-subtracted, high-background-screened and exposureand source-corrected images (maps) of the particle and photon components of the EPIC background have been created separately for each EPIC instrument, and in several different energy bands. This has been performed separately per observation, over a large number of individual observations.

The individual background maps for a particular instrument and in a particular energy band have then been collected together (for the same instrument mode/filter combination) over the whole set of observations. Via various cleaning, filtering and " $\sigma$-clipping" techniques, a "mean" background 
Table 2. The coordinate systems of the $1^{\prime}$ background detector maps and the 4 " detector maps used in the analysis.

\begin{tabular}{cccccc}
\hline \hline Instr. & $\begin{array}{c}\text { Coord. } \\
\text { (DETX/Y) }\end{array}$ & \multicolumn{2}{c}{ Range in } & \multicolumn{2}{c}{ No. of pixels } \\
min. & max. & $\left(4^{\prime \prime}\right)$ & $\left(1^{\prime}\right)$ \\
\hline PN & DETX & -19199 & 14400 & 420 & 28 \\
& DETY & -16799 & 15600 & 405 & 27 \\
M1 & DETX & -20399 & 20400 & 510 & 34 \\
& DETY & -20399 & 20400 & 510 & 34 \\
M2 & DETX & -20399 & 20400 & 510 & 34 \\
& DETY & -20399 & 20400 & 510 & 34 \\
\hline
\end{tabular}

map is created (for each particular background component/instrument/energy band/mode/filter combination).

Procedures have been written to perform the different aspects of the analysis, making extensive use of the XMM-SAS tasks, Chandra CIAO tools and HEASOFT's FTOOLS utilities.

Before discussing the analysis in depth, we describe some of the files and terms used, and the structure of some of the final products.

\subsection{Data analysis: Files and definitions}

We first define a number of terms used below:

- Background maps. Detector maps of all the background components combined i.e. soft and hard X-ray photons (vignetted), internal particles (non-vignetted), some small component of soft protons (scattered/funnelled) and singlereflections from out-of-FOV sources.

- Detector maps. Images in detector coordinates (DETX DETY) (as opposed to sky coordinates), defined, in the present analysis, such that the individual pixels are $1^{\prime}$ by $1^{\prime}$, DETX/DETY of $(0,0)$ lies at a pixel intersection, and the full area of the CCDs is covered. A scale of 1 arcmin has been chosen so as to give us decent count statistics in each pixel over the full energy band. More detailed information as to the coordinate system used (and the 4 " detector maps used within the analysis) is given in Table 2. Software has been written (see Sect. 4.2) to rebin the detector maps to any spatial scale, and to reproject the maps to any sky coordinates (specified via a user-input sky image).

- Instruments. The present analysis has been performed for each of the EPIC instruments; pn, MOS1 \& MOS2 (hereafter PN, M1, M2).

- Instrument mode \& filter. Several different data acquisition modes exist for each of the EPIC instruments. Also, observations have been taken using different filters for each of the EPIC instruments. The present analysis has been performed for the most common instrument mode/filter combinations, and those most useful to the analysis of diffuse $\mathrm{X}$-ray emission from extended objects. These are full-frame mode with thin filter (hereafter $\mathrm{ft}$ ) and full-frame mode with medium filter (fm), for each of PN, M1 \& M2, and also (for PN) full-frame-extended mode with thin filter (et) and fullframe-extended mode with medium filter (em).
- Energy bands. The analysis has been performed in the following XMM-XID \& PPS standard energy bands; energy bands 1-5, defined as follows; 1: 200-500 eV 2: $500-2000 \mathrm{eV}$ 3: $2000-4500 \mathrm{eV}$ 4: $4500-7500 \mathrm{eV}$ 5: 7500-12000 eV, and a full energy band 0: 200-12000 eV.

- Closed datasets. A few XMM observations have been obtained with the filter wheel in the "closed" position. No photons reach the CCDs, so the event files contain only the instrumental and particle components of the background. Such datasets have been collected together and processed, and exist on ftp://www-station.ias.u-psud.fr/ pub/epic/Closed (Marty et al. 2002). They are dependent on the instrumental mode, and have been processed for M1 full-frame (exposure $110655 \mathrm{~s}$ ), M2 full-frame (101336s), PN full-frame (42079s) and PN full-frameextended mode (26731 s).

\subsection{Data analysis: Preparation and reduction}

The initial analysis is essentially the same for each observation, and is detailed in the following steps:

- For each observation, the relevant Pipeline Processing System (PPS) products (event lists, source lists, attitude files, housekeeping files etc.), from the standard analysis carried out at the SSC, are collected together.

- For each instrument, region files are created from the PPS source lists. These are then used to remove all the source events from each of the relevant event files. A conservative extraction radius of $36^{\prime \prime}$ is used to remove the sources (for comparison, Lumb et al. 2002 used 25"). These regions are also removed from previously created mask files (these are required to calculate losses in area due to source removal).

- A visual inspection is made of the data to make sure that there are no strange features in the field, and to ascertain whether there are any very bright point sources or large diffuse sources which could contaminate the background, even after source subtraction. Datasets which fail this inspection are rejected from any subsequent analysis.

- Each of the event files are then filtered for periods of high background (solar proton flares). Lightcurves are created using the whole detector, for single events in the energy range $10-15 \mathrm{keV}$ (where no source counts are expected, due to the very low effective area of the telecope) and with FLAG values as defined by \#XMMEA_EM or \#XMMEA_EP. E.g. for PN, using the following expression for the XMMSAS task evselect;

PI in [10000:15000] \&\&\#XMEA_EP \&\&PATTERN==0 Good Time Interval (GTI) files are created from these lightcurves by applying an upper count rate threshold of $100(\mathrm{PN})$ or $35(\mathrm{M} 1 / \mathrm{M} 2) \mathrm{ct} / 100 \mathrm{~s}$. The event files are then filtered, keeping the low count rate time periods. The amount of time lost due to periods of high background flaring can be seen in Fig. 1, where the distribution of the fraction of data left after flare-screening is shown for all the datasets analysed. 


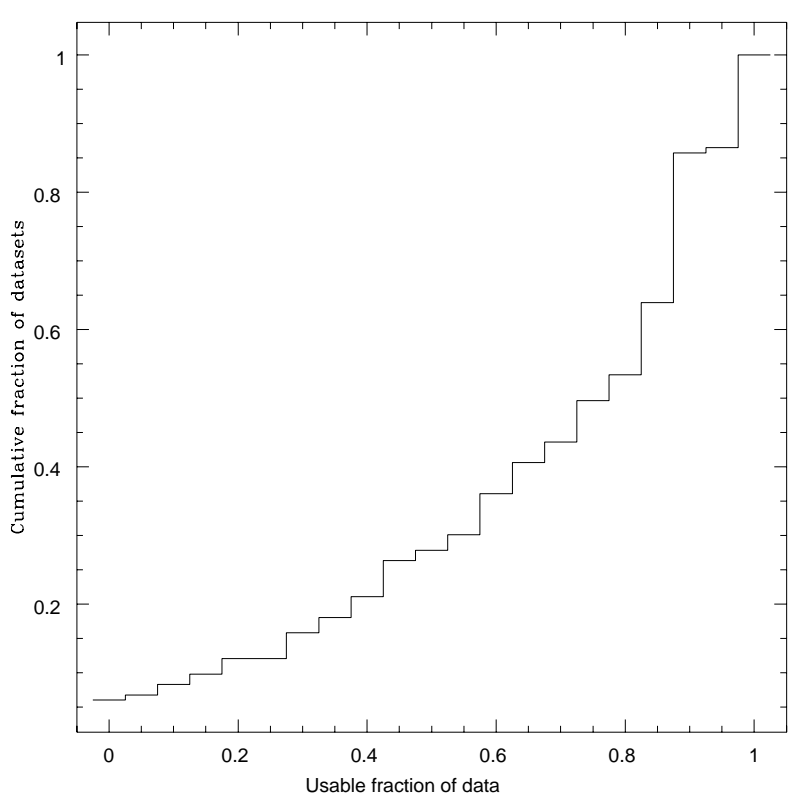

Fig. 1. The effects of high background flaring: The usable fraction of data available after flare-screening is shown for all the datasets analysed.

- The event files are then filtered further. Events with energies below $150 \mathrm{eV}$ are discarded. For PN, only singles and doubles are retained, for M1/M2, singles, doubles, triples and quadruples are retained. Finally, the event lists are filtered using the \#XMMEA_EM/P FLAG expressions, excepting that events from outside the field of view (out-of-FOV) are kept.

- In the case of PN, it was necessary to further filter the files to eliminate a small number of persistent bad (bright) pixels/columns, occurring in many (though not all) of the observations. The same pixels were also found to contaminate the PN closed datasets. These pixels were removed from all the PN datasets. Given the large-scale $\left(1^{\prime}\right)$ of the final BG maps, this had a very small effect, as the loss in area is very small. Nevertheless, this difference was corrected for in the final maps. The pixels removed were as follows: CCD1 Col. 13 \& (56, 75), CCD2 (46-47, 69-72), CCD5 Col. 11, CCD7 Col. 34, CCD10 Col. 61, CCD11 (47-48, 153-156) $(50,161-164)$. No bad pixels were removed from any of the MOS datasets (pointed observations or closed datasets). The event files are now filtered and have had all sources removed.

- For each of the three instruments, a small-scale (4") nonvignetted exposure map (with dimensions given in Table 2) is created. From the source-removed mask file, an area map ( $\left.4^{\prime \prime}\right)$ is created, containing zero values at the positions where sources have been removed, and unity values elsewhere. These two maps are multiplied and rebinned to form a large-scale ( $1^{\prime}$; see Table 2$)$ "area-times-exposure" map (see Fig. 2).

- For each instrument (of 3) and each energy range (of 6), $\left(1^{\prime}\right)$ detector maps are created. Because X-ray photons cannot reach the out-of-FOV areas of the detector, the events in these areas of the detector are solely due to the instrumental and particle components of the background. By making use of the Closed datasets, and comparing the number of counts in the out-of-FOV regions of the Closed datasets and the particular observation dataset in question, one can separate (for each mode-dependent instrument and energy range) the $1^{\prime}$ detector map into a particle (pa) map, containing the particle and instrumental background components and a photon (ph) map, containing the photon component of the background. This is done, producing 2 maps for each instrument and each energy range.

- The particle images are then exposure-corrected via a direct division by the LIVETIME (corrected for periods of high background and deadtime, deadtime being times when detector areas are affected by cosmic rays, and therefore not available to detect X-rays), and a division by a $1^{\prime}$ detector mask of the sensitive area of the CCDs (to correct for chip gaps). The photon images are exposure-corrected via a division by the appropriate "area-times-exposure" map.

\subsection{Data analysis: Observation, mode and filter selection}

The above preparatory analysis has been performed for 116 XMM-Newton observations, spanning a range in instrumental modes, filters, exposure times, and degree and duration of high-background flaring.

In order to produce the final background maps, the observations have been grouped together in terms of instrument/mode/filter etc. We then discarded datasets suffering from any of the following deficiencies:

- All observations containing a significantly bright source whose wings could still contaminate the background after source subtraction.

- All observations containing a large diffuse source, which could contribute to the estimated background.

- All observations where the background flaring was of such an extent that, after flare-removal, less than $10 \%$ of the original exposure remained.

The remaining 72 "clean" observations used in the production of the final background files are summarized in Table 3, which lists the revolution number, the RA and Dec, the Galactic hydrogen column, and a code giving the instrumental mode and filter for, respectively, M1, M2 and PN [ $\mathrm{f}$ - full-frame mode, e - full-frame-extended mode, $\mathrm{t}$ - thin filter, $\mathrm{m}$ - medium filter]. Also given are a nominal M1 exposure time, and the fraction of the exposure time remaining after removal of high-background periods and accounting for deadtime effects (here the PN fraction is given).

It is instructive to study some properties of the 72 clean observations. Figure 3 shows the distributions of (from left to right) the revolution number of the observation, the Galactic hydrogen column density, and the live exposure time, after cleaning for times of high background.

Almost all of the observations were taken in a 100-revolution period between the end of June 2001 and the 

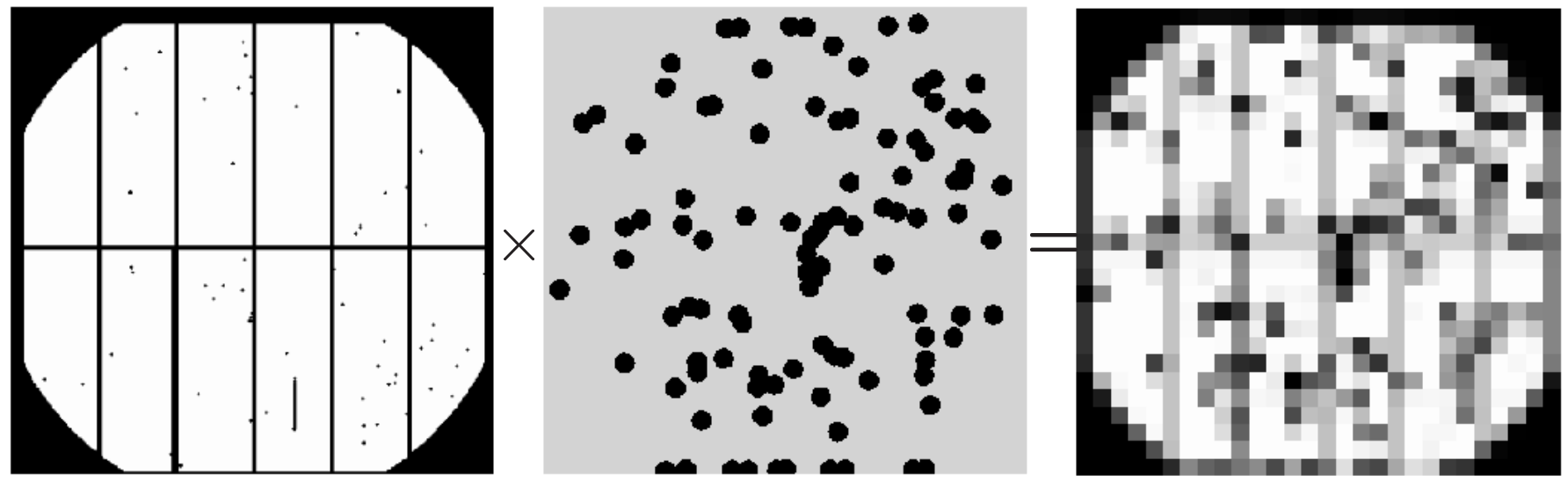

Fig. 2. A non-vignetted (4") exposure map is multiplied by a source-removed (4") mask map and rebinned to create a large-scale (1') "areatimes-exposure" map (example is for PN).
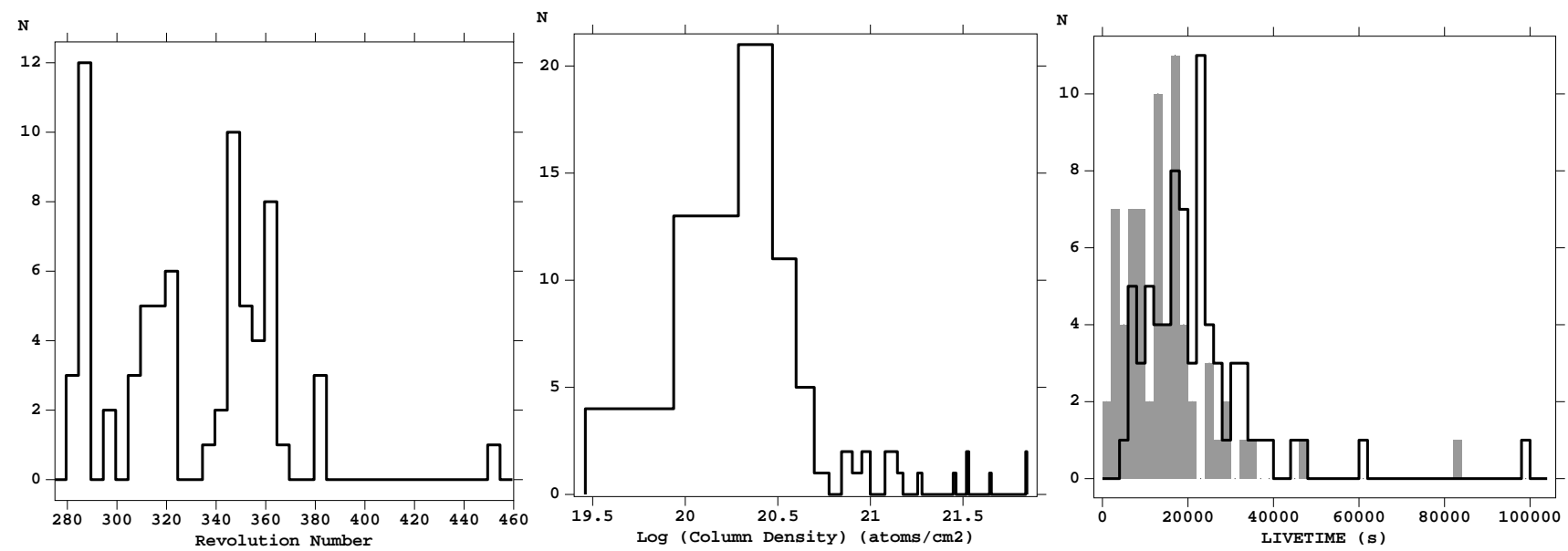

Fig. 3. Histogram distributions for the 72 clean observations of (from left to right) the observation revolution number, the Galactic hydrogen column, and the live exposure time (after cleaning for high background times) for M1 (thick line) and PN (filled region).

middle of January 2002. The Galactic hydrogen column density distribution shows that the majority of the observations are pointed in directions of low to medium column. A very small number of observations are pointed in directions of very high column. The post-flare-removal exposure-weighted mean value of the hydrogen column over all the 72 observations is $6.83 \times 10^{20} \mathrm{~cm}^{-2}$. The post-flare-removal exposure times show a systematically larger exposure time for the MOS instruments than for the pn (the M2 distribution is essentially identical to the M1 distribution shown). This is partly due to the pn being slightly more sensitive to proton flaring, but is mainly due to the fact that operational overheads generally lead to the pn having shorter exposure times (by $\approx 5 \mathrm{ks}$ ) than the MOS. Mean values of the live post-flare-removal exposure times are: M1: $22.0 \mathrm{ks}$ (standard deviation $13.8 \mathrm{ks}$ ), M2: $22.2 \mathrm{ks}$ (s.d. $13.8 \mathrm{ks}$ ), PN: $14.8 \mathrm{ks}$ (s.d. $11.9 \mathrm{ks}$ ).

Table 4 summarizes the final cleaned observation information in terms of the different combinations of instrument, instrument mode and filter used. The nominal exposure time is the sum of the individual LIVETIMEs i.e. corrected for periods of high-background and deadtime (see however the discussion of the exposure maps in Sect. 5.1).

\subsection{Data analysis: Cubes and clipping}

Here we describe the production of a single "averaged" background map from many background maps over different observations. At any given position in DETX/Y coordinates, a subset of the datasets used may be affected by contamination or diffuse sources. Such effects can be removed by clipping outliers, before taking the mean of the remaining "good" images at this position.

In order to produce a particular set of background maps (in the different energy bands) for a particular instrument/mode/filter combination, the following steps are taken. Let us take for example the first entry in Table 4, i.e. MOS1, full-frame mode, thin filter.

- For each of the 49 relevant observations, comprising in total over $1 \mathrm{Msec}$ of clean, low-background data, the 0-band (full energy band) $1^{\prime}$ photon detector maps of the background are stacked together into a 3D "imagecube" (see Fig. 4) of dimensions DETX, DETY (each in the case of MOS1 of size 34; see Table 2) and $N_{\text {obs }}$ (of size, in this example, 49).

- For each DETX/DETY, a statistical analysis of the (in this case, 49) values at that particular DETX/DETY point is performed. Here, a "clipcube" is constructed (with dimensions 
Table 3. Summary of the final cleaned and filtered observations used in the production of the EPIC background files. Given is the revolution number, the J2000.0 equinox RA and Dec, and the Galactic hydrogen column (in units of atoms $\mathrm{cm}^{-2}$ ) towards these coordinates. Also given is a code giving the instrumental mode and filter for M1, M2 and PN [ $\mathrm{f}$ - full-frame mode, $\mathrm{e}$ - full-frame-extended mode , $\mathrm{t}-$ thin filter, $\mathrm{m}-$ medium filter], plus the nominal exposure time, and the fraction of the pn exposure time remaining after removal of high-background periods.

\begin{tabular}{|c|c|c|c|c|c|c|c|c|c|c|c|c|c|}
\hline$\overline{\mathrm{Rev}}$ & $\begin{array}{l}\text { RA } \\
(20\end{array}$ & $\begin{array}{l}\text { Dec } \\
0.0)\end{array}$ & $\begin{array}{c}n \mathrm{H} \\
10^{20}\end{array}$ & $\begin{array}{l}\text { Mode/Filt. } \\
\text { M1M2PN }\end{array}$ & $\begin{array}{r}\text { Exp. } \\
\text { (s) }\end{array}$ & $f(\mathrm{Ex})$ & $\overline{\mathrm{Rev}}$ & $\begin{array}{l}\text { RA } \\
(20\end{array}$ & $\begin{array}{l}\text { Dec } \\
0.0)\end{array}$ & $\begin{array}{c}n \mathrm{H} \\
10^{20}\end{array}$ & $\begin{array}{l}\text { Mode/Filt. } \\
\text { M1M2PN }\end{array}$ & $\begin{array}{r}\text { Exp. } \\
\text { (s) }\end{array}$ & $\overline{\overline{f(\mathrm{Ex})}}$ \\
\hline 281 & 164.26 & -3.65 & 3.57 & $\mathrm{ft} \mathrm{ft}$ et & 40260 & 0.61 & 324 & 81.26 & -33.69 & 2.22 & $\mathrm{ft} \mathrm{ft} \mathrm{ft}$ & 27786 & 0.65 \\
\hline 283 & 16.93 & -17.49 & 1.53 & fmfmfm & 11813 & 0.13 & 339 & 125.14 & 21.10 & 4.23 & fmfmfm & 21617 & 0.88 \\
\hline 284 & 20.15 & -10.94 & 3.28 & $\mathrm{ft} \mathrm{ft} \mathrm{ft}$ & 9900 & 0.40 & 343 & 103.37 & -23.69 & 18.7 & fmfmem & 47559 & 0.37 \\
\hline 285 & 157.69 & -46.36 & 14.9 & fmfmem & 9215 & 0.89 & 344 & 121.11 & 65.02 & 4.32 & fmfmfm & 22615 & 0.73 \\
\hline 286 & 187.81 & 20.77 & 2.01 & $\mathrm{ft}$ fmet & 24211 & 0.81 & 345 & 144.97 & 35.93 & 1.43 & fmfmem & 34326 & 0.45 \\
\hline 286 & 187.81 & 21.27 & 1.84 & $\mathrm{ft}$ fmet & 24211 & 0.89 & 345 & 121.38 & 24.86 & 3.84 & $\mathrm{ft} \mathrm{ft}$ et & 24326 & 0.70 \\
\hline 286 & 187.81 & 21.77 & 1.76 & $\mathrm{ft}$ fmet & 24214 & 0.66 & 346 & 94.45 & -32.89 & 4.19 & $\mathrm{ft} f \mathrm{ft}$ et & 11773 & 0.90 \\
\hline 287 & 35.81 & -4.82 & 2.65 & $\mathrm{ft} f \mathrm{ft}$ et & 24218 & 0.89 & 346 & 93.85 & -33.39 & 3.43 & $\mathrm{ft} \mathrm{ft}$ et & 14772 & 0.46 \\
\hline 287 & 36.31 & -5.15 & 2.67 & $\mathrm{ft} \mathrm{ft}$ et & 24318 & 0.89 & 346 & 93.85 & -32.89 & 3.48 & $\mathrm{ft} \mathrm{ft}$ et & 14771 & 0.71 \\
\hline 287 & 35.98 & -5.15 & 2.64 & $\mathrm{ft} \mathrm{ft}$ et & 28512 & 0.88 & 346 & 127.91 & 52.78 & 3.85 & fmfmfm & 16916 & 0.90 \\
\hline 287 & 35.64 & -5.15 & 2.58 & $\mathrm{ft} \mathrm{ft}$ et & 24313 & 0.87 & 347 & 324.52 & -43.72 & 2.91 & $\mathrm{ft} \mathrm{ft} \mathrm{ft}$ & 7615 & 0.91 \\
\hline 288 & 352.95 & 19.65 & 4.19 & fmfmfm & 11513 & 0.77 & 348 & 154.66 & 41.45 & 1.12 & $\mathrm{ft} \mathrm{ft} \mathrm{ft}$ & 29617 & 0.76 \\
\hline 288 & 36.98 & -5.15 & 2.64 & $\mathrm{ft} \mathrm{ft}$ et & 25149 & 0.74 & 348 & 137.86 & 5.88 & 3.65 & $\mathrm{ft} f t$ et & 20072 & 0.22 \\
\hline 288 & 36.64 & -5.15 & 2.66 & $\mathrm{ft} \mathrm{ft}$ et & 12268 & 0.17 & 349 & 163.18 & 57.51 & 0.56 & fmfmfm & 37950 & 0.77 \\
\hline 288 & 14.69 & -27.58 & 1.91 & $\mathrm{ft} \mathrm{ft}$ et & 9209 & 0.62 & 353 & 162.47 & 33.01 & 1.98 & $\mathrm{ft} f t$ et & 37800 & 0.36 \\
\hline 299 & 53.08 & -27.79 & 0.90 & $\mathrm{ft} \mathrm{ft}$ et & 39765 & 0.49 & 353 & 152.79 & 55.78 & 0.78 & $\mathrm{xx} \mathrm{ft} \mathrm{ft}$ & 32615 & 0.83 \\
\hline 299 & 53.09 & -27.79 & 0.90 & $\mathrm{ft} \mathrm{ft}$ et & 56698 & 0.65 & 353 & 143.95 & 61.38 & 2.68 & fmfmet & 34371 & 0.89 \\
\hline 300 & 213.95 & 11.47 & 1.82 & $\mathrm{ft} \mathrm{ft}$ et & 25719 & 0.88 & 354 & 335.21 & -24.70 & 1.94 & fmfmfm & 27617 & 0.85 \\
\hline 305 & 222.38 & 8.97 & 2.03 & $\mathrm{ft} \mathrm{ft} \mathrm{ft}$ & 40387 & 0.86 & 354 & 314.11 & -4.65 & 4.97 & $\mathrm{xx}$ fmem & 16770 & 0.89 \\
\hline 307 & 201.70 & -47.50 & 9.54 & fmfmfm & 39978 & 0.47 & 355 & 333.91 & -17.75 & 2.36 & $\mathrm{ft} \mathrm{ft} x \mathrm{x}$ & 34397 & 0.96 \\
\hline 308 & 36.65 & -5.15 & 2.63 & $\mathrm{ft} \mathrm{ft}$ et & 12666 & 0.89 & 355 & 333.91 & -17.75 & 2.36 & $\mathrm{ft} \mathrm{ft} \mathrm{ft}$ & 10824 & 0.62 \\
\hline 310 & 64.90 & 56.02 & 44.6 & fmfmfm & 32310 & 0.40 & 356 & 333.90 & -17.75 & 2.36 & $\mathrm{ft} \mathrm{ft} \mathrm{ft}$ & 109427 & 0.77 \\
\hline 311 & 224.60 & -31.70 & 8.42 & $\mathrm{ft} \mathrm{ft} \mathrm{ft}$ & 52799 & 0.43 & 359 & 148.42 & 1.61 & 3.53 & $\mathrm{ft} f t$ et & 10020 & 0.39 \\
\hline 312 & 0.67 & 62.77 & 69.3 & fmfmsm & 33000 & 0.49 & 360 & 152.77 & -4.66 & 3.83 & fmfmem & 19130 & 0.21 \\
\hline 312 & 4.07 & 81.58 & 13.9 & fmfmfm & 39337 & 0.35 & 360 & 169.57 & 7.79 & 3.51 & fmfmft & 62616 & 0.78 \\
\hline 314 & 94.65 & 78.38 & 7.30 & fmfmft & 26847 & 0.40 & 360 & 182.38 & 43.71 & 1.35 & $\mathrm{ft} \mathrm{ft}$ em & 14317 & 0.87 \\
\hline 316 & 259.92 & -25.04 & 28.5 & $\mathrm{ft} \mathrm{ft} \mathrm{ft}$ & 12225 & 0.70 & 361 & 355.00 & -12.31 & 2.76 & $\mathrm{ft} \mathrm{ft}$ et & 14369 & 0.86 \\
\hline 316 & 253.52 & -39.88 & 69.1 & fmfmfm & 39820 & 0.46 & 361 & 339.27 & -15.29 & 3.89 & $\mathrm{ft} \mathrm{ft}$ et & 24370 & 0.78 \\
\hline 317 & 58.46 & 23.41 & 9.47 & $\mathrm{ft} \mathrm{ft} \mathrm{ft}$ & 34335 & 0.31 & 362 & 346.21 & 3.18 & 5.25 & $\mathrm{ft} \mathrm{ft} \mathrm{ft}$ & 12566 & 0.88 \\
\hline 317 & 51.39 & 30.75 & 13.8 & $\mathrm{ft} \mathrm{ft} \mathrm{ft}$ & 32725 & 0.32 & 363 & 159.97 & 20.87 & 2.02 & $\mathrm{ft} \mathrm{ft}$ et & 14365 & 0.47 \\
\hline 317 & 76.31 & -28.80 & 1.49 & $\mathrm{ft} f \mathrm{ft}$ et & 48870 & 0.64 & 364 & 183.06 & 13.23 & 2.57 & $\mathrm{ft} \mathrm{ft}$ em & 22867 & 0.86 \\
\hline 321 & 250.21 & -24.13 & 12.5 & $\mathrm{ft} f \mathrm{ft}$ et & 20121 & 0.88 & 366 & 231.50 & 51.64 & 1.56 & $\mathrm{ft} \mathrm{ft} \mathrm{ft}$ & 36926 & 0.72 \\
\hline 321 & 250.21 & -24.43 & 12.4 & $\mathrm{ft}$ fmet & 18626 & 0.82 & 383 & 36.02 & -3.85 & 2.49 & $\mathrm{ft} f \mathrm{ft}$ et & 13382 & 0.85 \\
\hline 322 & 79.07 & 79.69 & 7.99 & fmfmfm & 31997 & 0.90 & 383 & 35.52 & -3.52 & 2.48 & $\mathrm{ft} \mathrm{ft}$ et & 13382 & 0.44 \\
\hline 323 & 263.57 & -26.11 & 33.3 & $\mathrm{ft} \mathrm{ft} \mathrm{ft}$ & 24618 & 0.61 & 383 & 35.86 & -3.52 & 2.52 & $\mathrm{ft} \mathrm{ft}$ et & 12380 & 0.87 \\
\hline 323 & 263.57 & -26.11 & 33.3 & $\mathrm{ft} \mathrm{ft} \mathrm{ft}$ & 25115 & 0.60 & 451 & 187.65 & 41.62 & 1.78 & fmfmem & 17521 & 0.85 \\
\hline
\end{tabular}

identical to the input imagecube), containing information (1's and 0's) as to which cells in the imagecube are within the allowed range and which values are not, i.e. which values are "clipped" (see Fig. 5). The allowable range is defined as within some number of standard deviations $(\sigma \mathrm{s})$ from the mean value at that DETX/DETY. For the present analysis, this number of $\sigma \mathrm{s}$ is set at 1.2. Steps are taken, including the prior removal of very strongly aberrant pixels, to ensure that the initial mean used to define the clip limits is not seriously biased by outliers. The mean value at that particular DETX/DETY is then calculated from the remaining " $\sigma$-clipped" values.

- Imagecubes are then created, as in the 0-band, from the intermediate energy band (bands 1-5) 1' photon detector maps of the background. Due to the limited statistics in the band 1-5 imagecubes, the broad-band clipcube is used to create the $\sigma$-clipped band $1-5$ images. The same procedure is performed for the particle images, and for all instrument/mode/filter combinations.

\section{The background maps}

$\sigma$-clipped, and averaged, exposure-corrected background maps have been created as described above for each instrument/mode/filter combination analysed (of 8) and in each energy band (of 6). Photon and particle maps have been created separately, as have maps with the two components recombined. This leads to a total of 144 background maps, and these are shown in Figs. 6-9. The maps can be obtained from http://www.sr. bham.ac.uk/xmm3/.

A1_ftQ000_cphim4M1.fits is, as an example of the file naming, an exposure-corrected photon background map. A particle map is named *cpaim*, instead of *cphim*, and a 
Table 4. Summary of the cleaned and filtered observations used in the production of the EPIC background files, separated into the different combinations of instrument, instrument mode and filter used. The exposure time is the sum of the individual LIVETIMEs i.e. corrected for periods of high-background and deadtime. The post-flare-removal exposure-weighted mean value of the hydrogen column is also tabulated.

\begin{tabular}{lllrrr}
\hline \hline Instrument & Mode & Filter & $\begin{array}{r}\text { Number of } \\
\text { Observations }\end{array}$ & $\begin{array}{r}\text { Exposure } \\
\text { Time }(\mathrm{s})\end{array}$ & $\begin{array}{r}n \mathrm{H} \\
10^{20} \mathrm{~cm}^{-2}\end{array}$ \\
\hline MOS1 & Full-Frame & Thin & 49 & 1055905 & 4.49 \\
MOS1 & Full-Frame & Medium & 21 & 488422 & 12.7 \\
MOS2 & Full-Frame & Thin & 46 & 1004709 & 4.40 \\
MOS2 & Full-Frame & Medium & 26 & 592975 & 11.1 \\
PN & Full-Frame & Thin & 18 & 351549 & 6.35 \\
PN & Full-Frame & Medium & 12 & 188159 & 13.3 \\
PN & Full-Frame-Extended & Thin & 32 & 416739 & 3.05 \\
PN & Full-Frame-Extended & Medium & 8 & 82957 & 6.13 \\
\hline
\end{tabular}

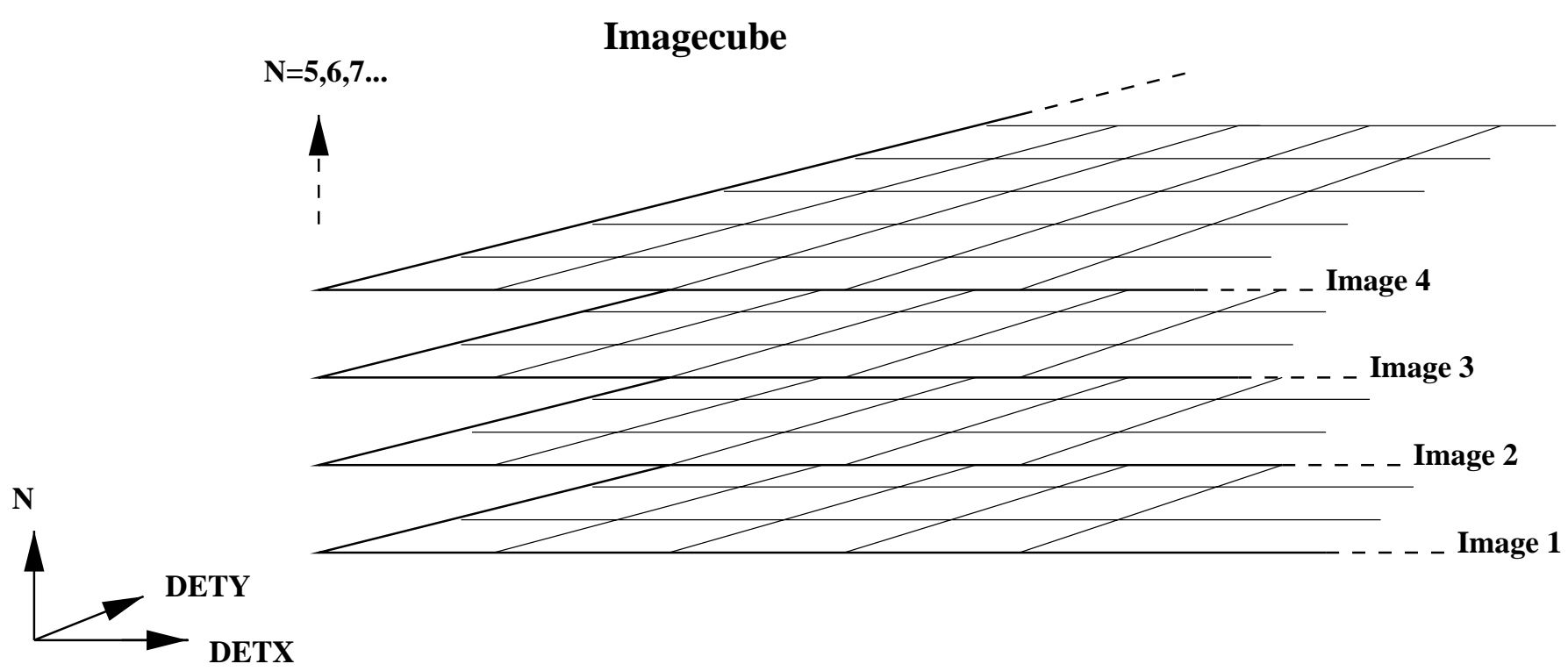

Fig. 4. A 3D "imagecube" of dimensions DETX, DETY and $N_{\text {obs. }}$.

particle and photon combined map *cim*. The example above is a MOS1 map (M1 instead of M2 or PN), and is in energy band 4 (of $Q-6$ ). The mode is full-frame (given by the $\mathrm{f}$ ), as opposed to (for PN) extended full-frame mode (e), and the filter is thin $(\mathrm{t})$, as opposed to medium (m). The six character mode+filter string is as in Table 3, hence the corresponding PN file is named A1_0000ft_cphim4PN.fits. The A1 indicates a first release of these background maps.

\subsection{The background maps: Usage}

When might an observer with their own EPIC data want to make use of these background maps? In order to analyse extended objects, and make background-subtracted images, estimate low-surface brightness flux levels, create radial profiles or perform 1- or 2D surface brightness fitting, one needs a map of the appropriate background. Sometimes however, the diffuse, extended nature of the user's target source is such that the determination of the background from their own dataset is difficult or impossible. Also, using a background from a significantly spatially removed section of the same data leads to problems with vignetting and detector variations. Hence the need for the independently-produced background maps created here.
What follows is a recipe for how an observer can make use of the background maps in conjunction with their own source data. Caveats, and problems that can occur when the recipe is not or can not be followed are given thereafter.

- The source data should be flare-rejected to a similar level to that applied in the creation of the background maps ${ }^{1}$.

- Images should be created from the flare-rejected source datasets in the same XID/PPS energy bands (bands 0-5) as used here ${ }^{2}$.

- As a particular source image will be created (most commonly) in sky coordinates, and will have a resolution finer than $1^{\prime}$, the routine BGrebinimage $2 S K Y$ should be used to rebin and sky-project the equivalent background map(s) to the resolution and sky position of the source image (see Sect. 4.2).

- Depending on whether the source maps are raw count maps or exposure-corrected flux maps, the equivalent rebinned background map(s) (which are exposure-corrected) may be scaled appropriately, either by an exposure time, or using an appropriate exposure map.

- A comparison of the counts or flux in the out-of-FOV areas of the source image and background map yields the scaling 


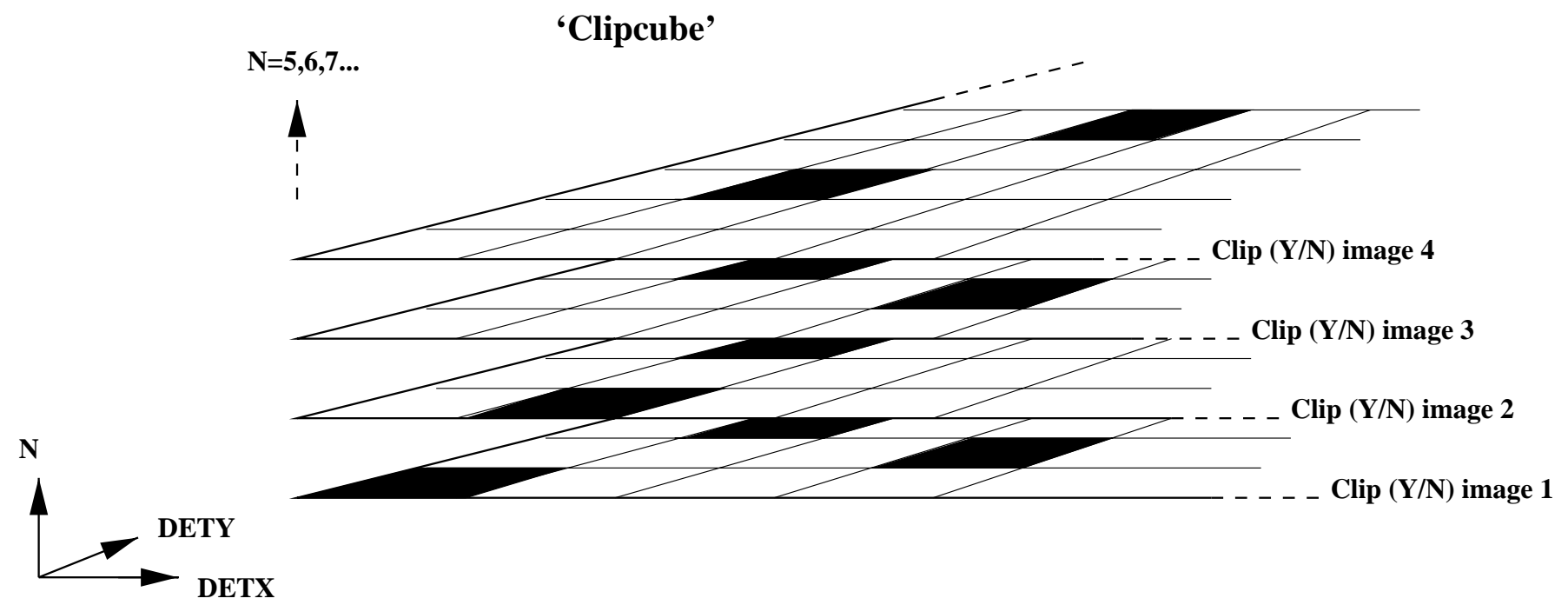

Fig. 5. A 3D "clipcube" of dimensions DETX, DETY and $N_{\text {obs. }}$ In this example, for DETX/DETY $=(1,1)$, the value in image 1 is outside of the allowable range, and is to be clipped from the calculation of the mean value for DETX/DETY $=(1,1)$

by which the particle component (the "cpaim" map) of the background needs be scaled ${ }^{3}$.

- If source-free regions within the FOV exist within the source dataset, then count/flux comparisons in these regions yield the scaling for the photon component (the "cphim" map). The scaled photon component can then be add to the scaled particle component to give the final background map $^{3}$.

${ }^{1}$ If such a flare-rejection method as used here leads to zero or very few Good Times, then the user's data is heavily flarecontaminated, and the background maps are therefore not suitable for source data extracted from the whole dataset. Small discrepancies due to slightly different flare-rejection methods may be accounted for by the subsequent scaling.

2 Though the user should work using the same energy bands (bands 0-5) as used in the present analysis, note that the standard XID/PPS bands have been used, and that extension to larger energy bands can easily be performed by summing individual band images. For example, band 1 is heavily contaminated by detector noise in the particle background, so the user may prefer to work in band $(2+3+4+5)$.

${ }^{3}$ If source-free regions within the FOV do exist within the source dataset, then the user is advised to work as above with the particle and photon ("cpaim" \& "cphim") maps separately. The particle and photon scaling factors may be different (hence the fact that separate particle and photon background maps have been made available). If no source-free regions exist, the user may be forced to assume that the scalings are the same. Here, just the "cim" images need be used. A script for comparing out-of-FOV counts (from event files), compareoutof$F O V$, is available on http://www.sr.bham.ac.uk/xmm3/. Note especially that at the very edges of the map FOVs, there are a few pixels with unusually large and small values. This is due to extremely small exposure values in the original observation maps amplifying the noise. These areas should be avoided when calculating scaling values.

\subsection{The background maps: Statistics}

For each of the instrument/mode/filter combinations, and for each of the energy bands $0-5$, mean count rates (in units of ct $\mathrm{ks}^{-1} \operatorname{arcmin}^{-2}$ ) and standard deviations about these mean values have been calculated using all of the contributing observations listed in Table 3. This has been done separately for the photons and for particles. For the photons, the mean values were calculated within the central $16^{\prime} \times 16^{\prime}$, to avoid the small number of pixels discussed above with unusually large and small values. Table 5 lists these values for the photons, Table 6 lists them for the particles.

Several features are evident from the tables. For a particular instrument, the photon and particle background count rates are of the same order, the values for the pn being a factor of a few greater than for the MOS.

Whereas, for the photons, the decline in intensity (in ct ks ${ }^{-1} \operatorname{arcmin}^{-2} \mathrm{keV}^{-1}$ with increasing energy is quite steep, for the particles, it is far flatter, emphasizing the points made in Table 1, i.e. that the particle component of the background appears essentially flat, and dominates above $5 \mathrm{keV}$ over the photon $(\sim 1.4$ power law + soft thermal lines) component. This can be seen in Figs. 6-9 also; at low energies, the photon maps are brighter, whilst at higher energies, the particle maps dominate. In studying the actual values, one can see that, on average, for the MOS, the photons dominate the background over most of the spectrum, the particles only attaining a comparable count rate in the highest energy band. For the pn however, the particles attain a comparable count rate to the photons at a lower energy, and are then far more dominant at the highest energies. Again, this can be seen in Figs. 6-9.

The standard deviation in the mean values represents the scatter in the mean background count rates over the different observations. Note though there is likely some contribution from errors introduced in the separating of particles from photons, a large contributor being the presence of the extremely small exposure values in the original observation maps 

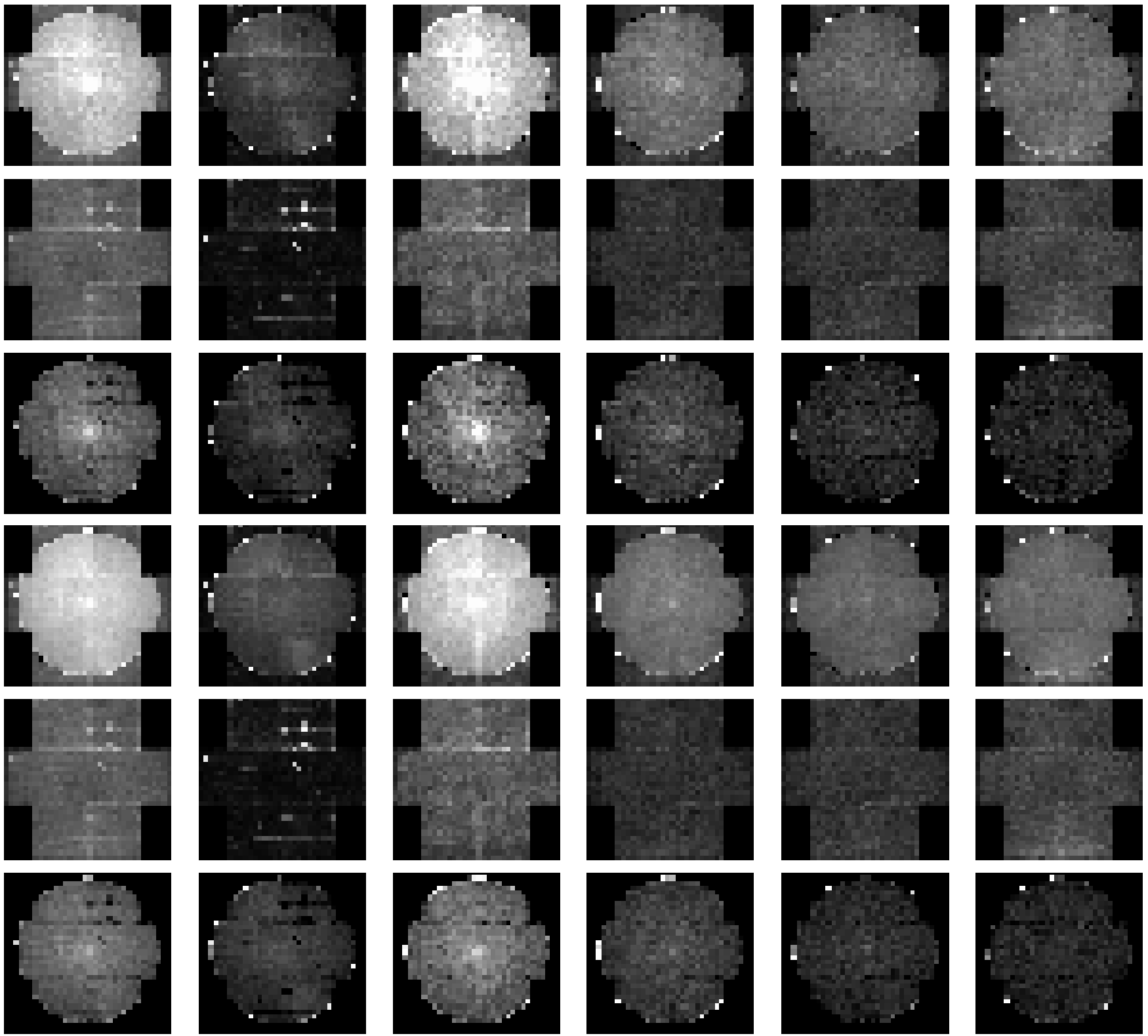

Fig. 6. The final $\sigma$-clipped, averaged, and exposure-corrected background maps, for MOS1, full-frame mode. Energy band 0 (full-band), then bands 1-5 run from left to right. Rows 1-3 are for medium filter and show (row 1) the particle and photon combined (cim) maps, (row 2) the particle (cpaim) maps, and (row 3) the photon (cphim) maps. Rows 4-6 show the equivalent maps for thin filter. All 0-band maps are to the same level of scaling. All other maps are to a scaling 1/3 that of the 0 -band maps. Note that at the very edges of the photon (and combined) map FOVs, there are sometimes a few pixels with unusually large or small values. This is due to extremely small exposure values in the original observation maps amplifying the noise.

amplifying the noise in the out-of-FOV regions. This effect is more prevalent in the pn datasets (where the out-of-FOV regions are smaller). Also, there will be some contribution to the scatter due to the Poissonian variation in the out-ofFOV counts used to normalise the particle contribution. For the lower-exposure datasets, this can approach $10 \%$ in some energy bands.

As regards the particles, the scatter seen in the MOS particle background rates is small, about $10 \%$, and appears roughly constant with energy. The pn experiences a larger scatter of about $20 \%$, and of this, the greatest scatter $(50 \%)$ is observed at lower energies. Not all of this scatter however, is thought due to variations in the actual particle count rates (see above). The photons show quite a range in count rate scatter (from $30-60 \%$ and beyond), with no particular trend with energy or instrument.

Figure 10 shows an example of the distribution of background count rates observed within the sample. Shown are (thick line) the M1 0-band full-frame thin filter photon count rate distribution [49 entries; Table 4] (the equivalent M2 distribution appears almost identical), and (filled region) the PN 0-band full-frame-extended thin filter photon count rate distribution [32 entries]. The pn distribution has a mean value 

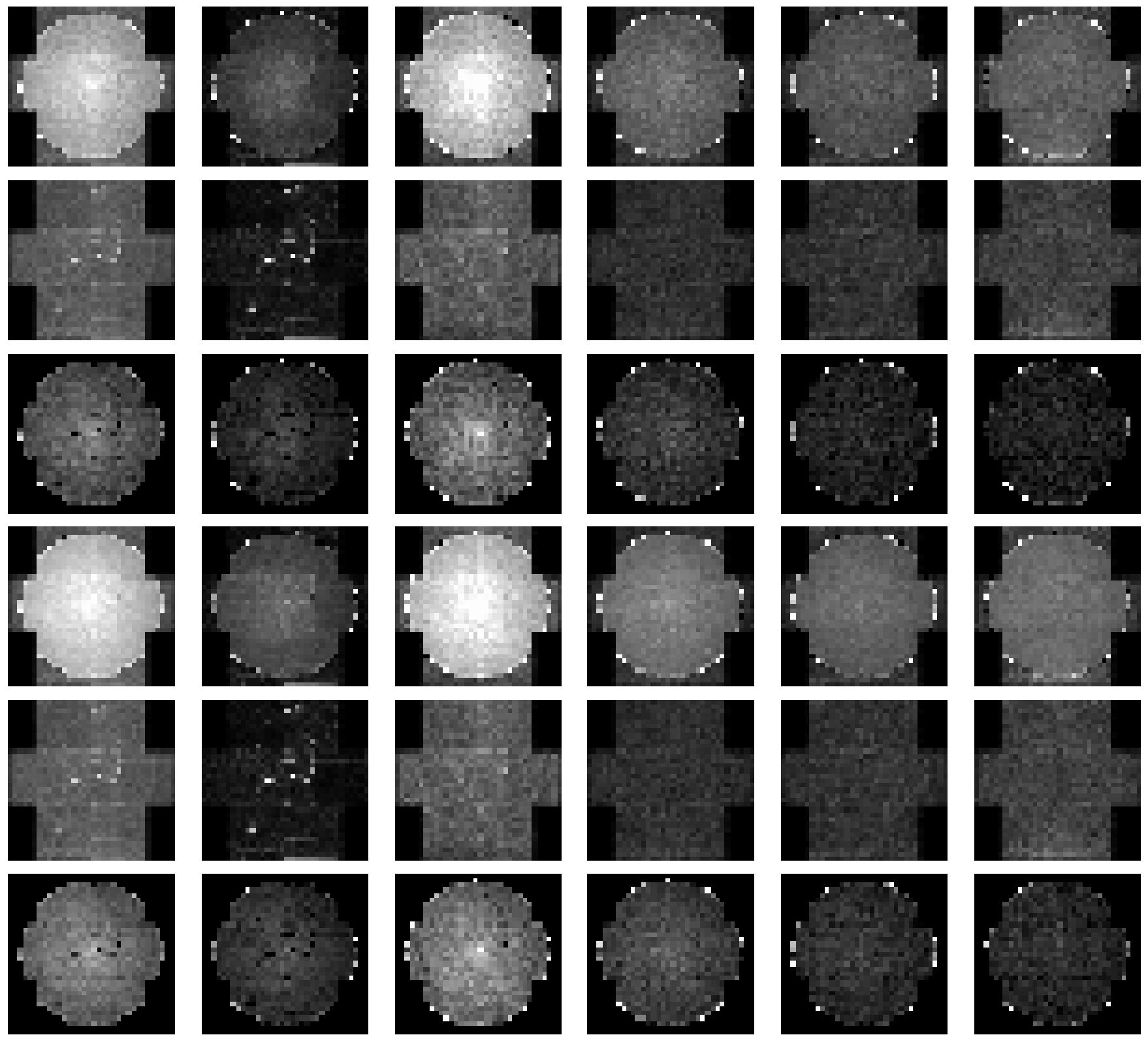

Fig. 7. The final $\sigma$-clipped, averaged, and exposure-corrected background maps, as for Fig. 6, but for MOS2, full-frame mode.

Table 5. Mean count rates for the photon background maps (over the central $16^{\prime} \times 16^{\prime}$ ), for the different instrument, mode and filter combinations, and for each of the six (five plus total) standard energy bands.

\begin{tabular}{llccccccc}
\hline \hline Instr. & Mode/ & $N_{\text {obs }}$ & \multicolumn{7}{c}{ Mean Count Rate $\left(\mathrm{ct} \mathrm{ks}^{-1} \operatorname{arcmin}^{-2}\right)(+$ standard deviation) } \\
& filter & & Band 0: & Band 1: & Band 2: & Band 3: & Band 4: & Band 5: \\
& & & $200-12000 \mathrm{eV}$ & $200-500 \mathrm{eV}$ & $500-2000 \mathrm{eV}$ & $2000-4500 \mathrm{eV}$ & $4500-7500 \mathrm{eV}$ & $7500-12000 \mathrm{eV}$ \\
\hline MOS1 & $\mathrm{ft}$ & 49 & $2.10(1.36)$ & $0.37(0.18)$ & $0.78(0.46)$ & $0.49(0.37)$ & $0.35(0.22)$ & $0.31(0.18)$ \\
MOS1 & $\mathrm{fm}$ & 21 & $2.00(1.06)$ & $0.32(0.12)$ & $0.80(0.38)$ & $0.48(0.27)$ & $0.33(0.16)$ & $0.29(0.14)$ \\
MOS2 & $\mathrm{ft}$ & 46 & $2.23(1.40)$ & $0.39(0.19)$ & $0.84(0.50)$ & $0.53(0.39)$ & $0.37(0.22)$ & $0.32(0.18)$ \\
MOS2 & $\mathrm{fm}$ & 26 & $1.81(1.07)$ & $0.31(0.12)$ & $0.76(0.39)$ & $0.42(0.27)$ & $0.29(0.16)$ & $0.27(0.13)$ \\
PN & $\mathrm{ft}$ & 18 & $6.50(3.89)$ & $1.90(1.02)$ & $2.46(1.28)$ & $0.93(0.71)$ & $0.69(0.43)$ & $0.61(0.24)$ \\
PN & $\mathrm{fm}$ & 12 & $4.31(2.24)$ & $1.13(0.50)$ & $2.04(0.94)$ & $0.72(0.33)$ & $0.64(0.36)$ & $0.68(0.48)$ \\
PN & et & 32 & $5.19(4.38)$ & $1.87(2.06)$ & $1.71(1.01)$ & $0.86(0.68)$ & $0.72(0.52)$ & $0.79(0.55)$ \\
PN & em & 8 & $5.41(2.66)$ & $1.94(0.77)$ & $1.90(0.47)$ & $0.90(0.60)$ & $0.76(0.50)$ & $0.74(0.40)$ \\
\hline
\end{tabular}



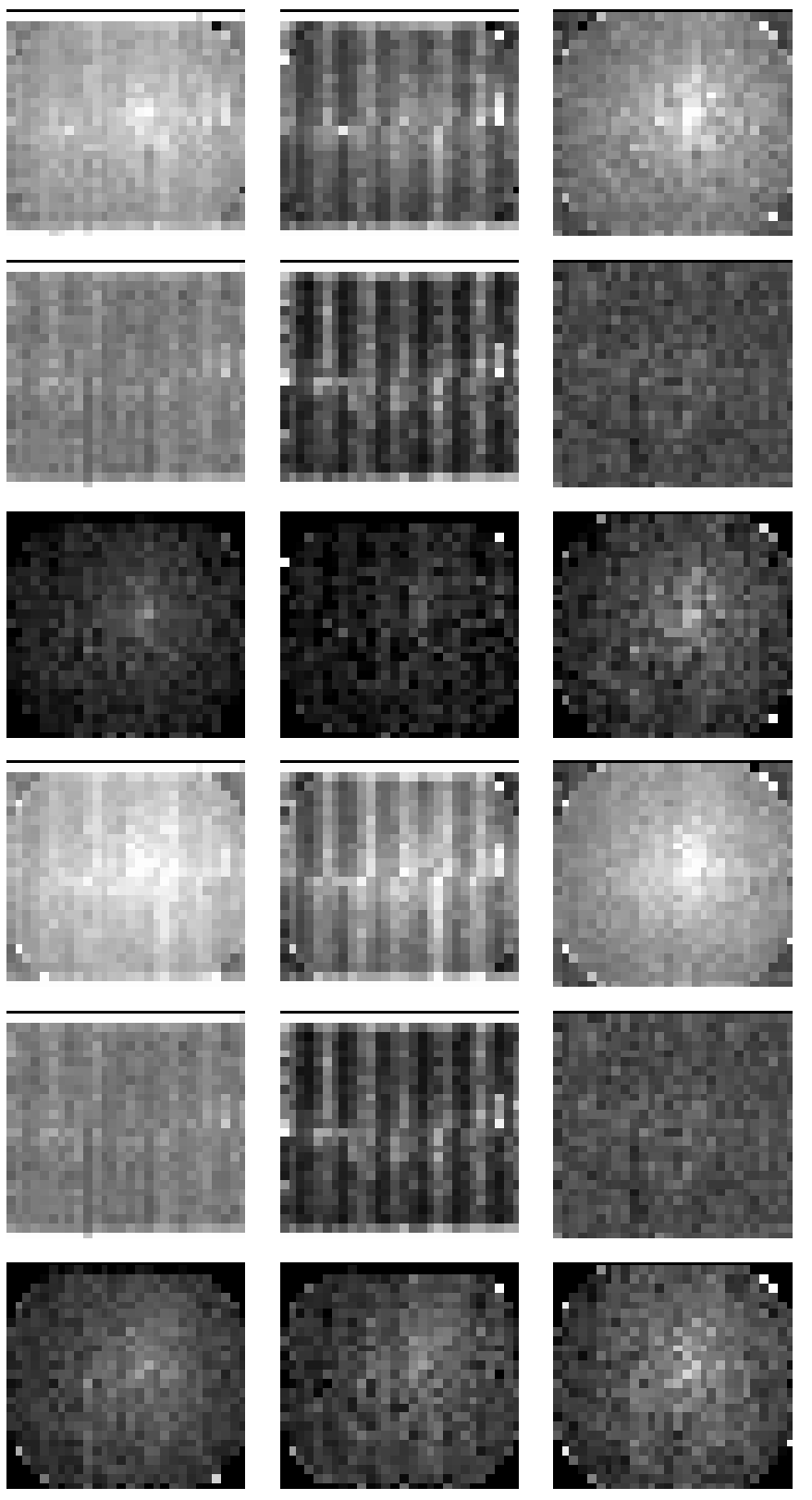
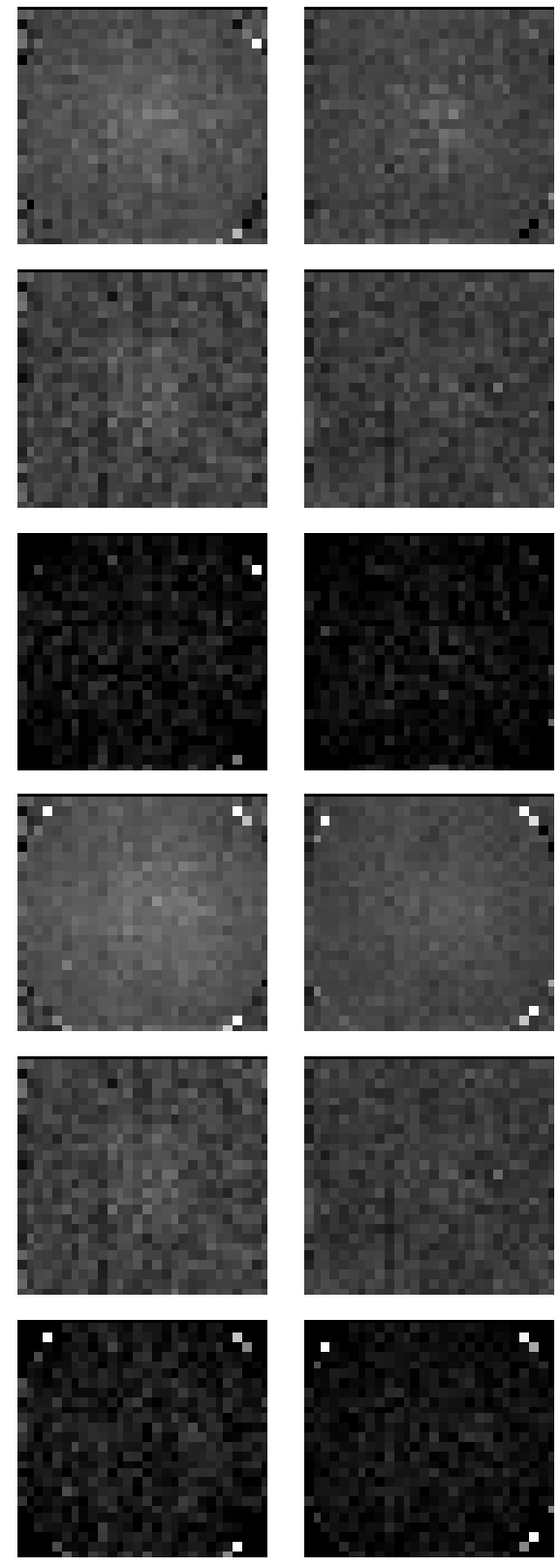
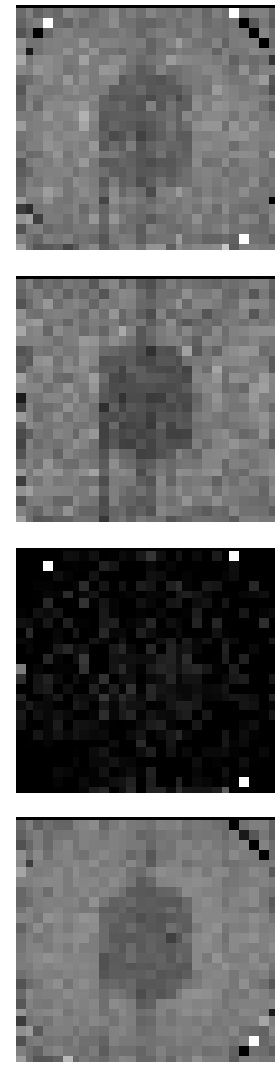

Fig. 8. The final $\sigma$-clipped, averaged, and exposure-corrected background maps, as for Fig. 6, but for pn, full-frame mode.

Table 6. Mean count rates for the particle background maps for the different instrument, mode and filter combinations, and for each of the six (five plus total) standard energy bands.

\begin{tabular}{llccccccc}
\hline Instr. & Mode/ & $N_{\text {obs }}$ & \multicolumn{7}{c}{ Mean Count Rate $\left(\mathrm{ct} \mathrm{ks}^{-1}\right.$ arcmin $\left.^{-2}\right)(+$ standard deviation) } \\
& filter & & Band 0: & Band 1: & Band 2: & Band 3: & Band 4: & Band 5: \\
& & & $200-12000 \mathrm{eV}$ & $200-500 \mathrm{eV}$ & $500-2000 \mathrm{eV}$ & $2000-4500 \mathrm{eV}$ & $4500-7500 \mathrm{eV}$ & $7500-12000 \mathrm{eV}$ \\
\hline \hline MOS1 & $\mathrm{ft}$ & 49 & $1.40(0.11)$ & $0.12(0.03)$ & $0.44(0.04)$ & $0.24(0.02)$ & $0.26(0.02)$ & $0.34(0.03)$ \\
MOS1 & $\mathrm{fm}$ & 21 & $1.43(0.11)$ & $0.13(0.03)$ & $0.45(0.04)$ & $0.24(0.02)$ & $0.26(0.02)$ & $0.34(0.02)$ \\
MOS2 & $\mathrm{ft}$ & 46 & $1.34(0.09)$ & $0.14(0.02)$ & $0.42(0.03)$ & $0.23(0.02)$ & $0.24(0.02)$ & $0.32(0.02)$ \\
MOS2 & $\mathrm{fm}$ & 26 & $1.31(0.09)$ & $0.13(0.02)$ & $0.42(0.04)$ & $0.23(0.02)$ & $0.24(0.02)$ & $0.31(0.02)$ \\
PN & $\mathrm{ft}$ & 18 & $8.37(2.29)$ & $2.13(0.43)$ & $1.95(1.36)$ & $1.50(0.86)$ & $1.13(0.31)$ & $2.05(0.21)$ \\
PN & $\mathrm{fm}$ & 12 & $8.16(1.60)$ & $2.23(0.31)$ & $1.55(0.77)$ & $1.32(0.47)$ & $1.11(0.20)$ & $2.08(0.25)$ \\
PN & et & 32 & $7.96(1.52)$ & $2.10(0.29)$ & $1.61(0.86)$ & $1.32(0.58)$ & $1.15(0.32)$ & $2.01(0.20)$ \\
PN & em & 8 & $8.22(2.72)$ & $2.27(0.87)$ & $1.58(0.81)$ & $1.31(0.64)$ & $1.12(0.31)$ & $2.05(0.26)$ \\
\hline
\end{tabular}



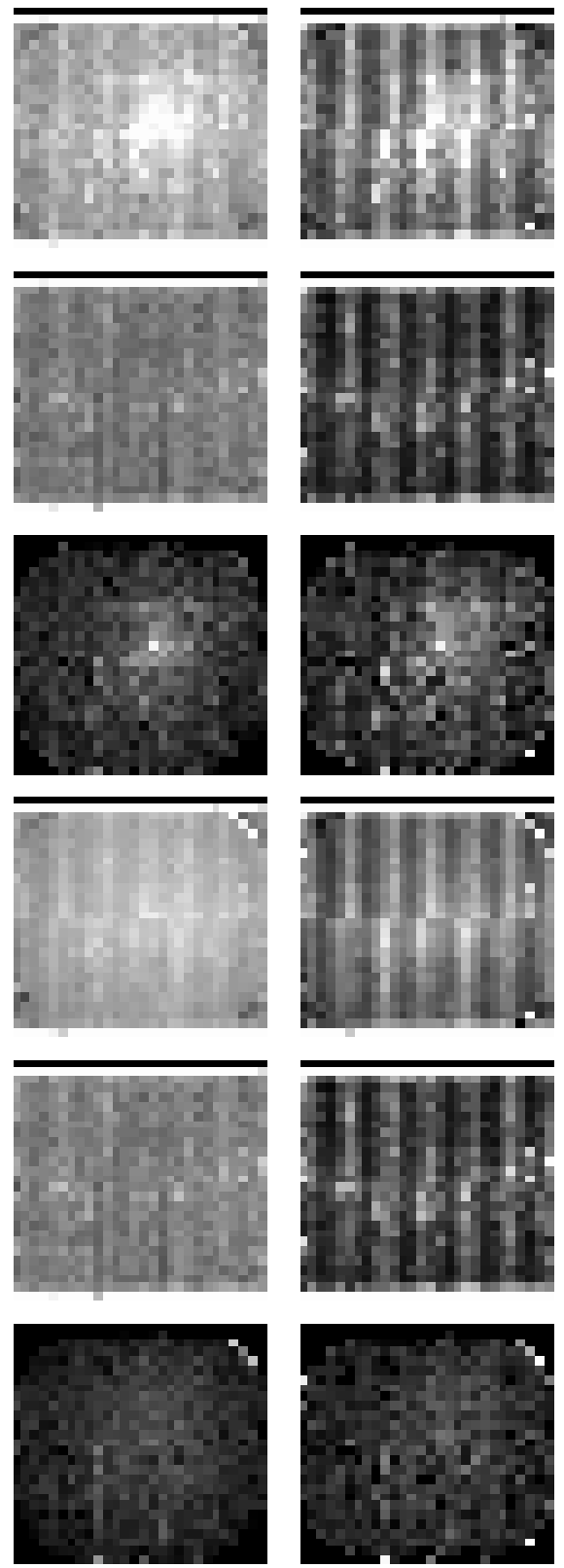
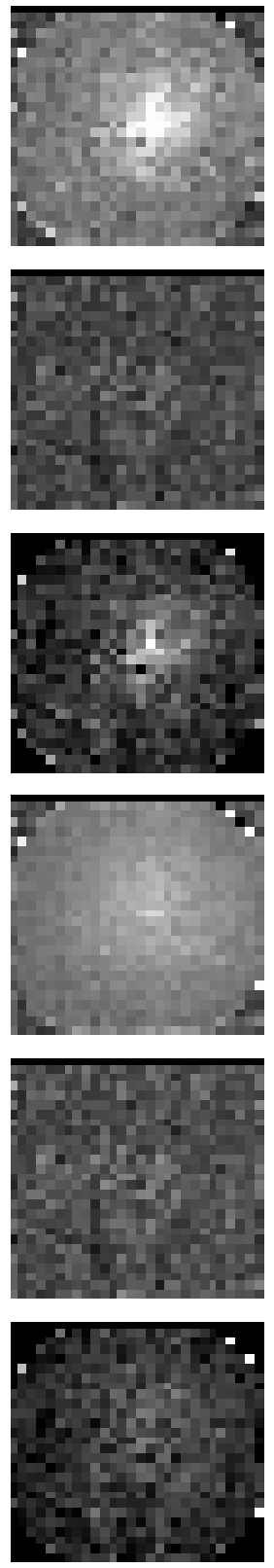
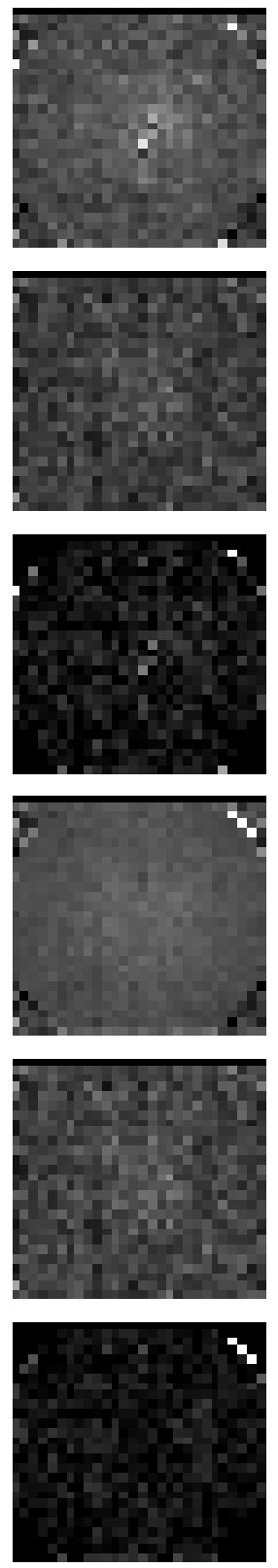
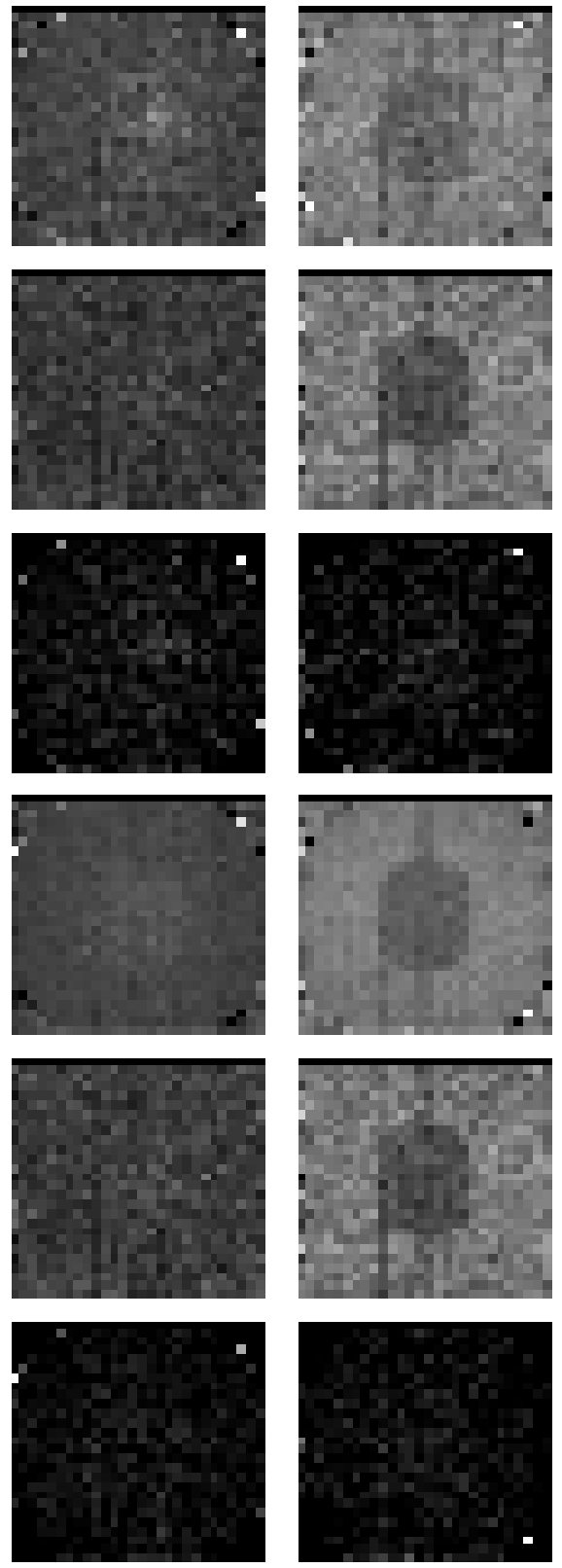

Fig. 9. The final $\sigma$-clipped, averaged, and exposure-corrected background maps, as for Fig. 6, but for pn, extended full-frame mode.

(in this example) some $\approx 2.5$ times that for the MOS, and has quite a larger scatter.

\subsection{The background maps: Sky coordinates, rebinning and reprojecting}

Usually, of course, the user will work in sky coordinates (RA and Dec), not detector coordinates, and to a much finer resolution than $1^{\prime}$. With that in mind, software has been developed to rebin and reproject onto the sky any provided background map (scaled or otherwise) to the spatial scale and sky position of a user-input image.

BGrebinimage2SKY is a shell script plus FORTRAN routine to convert the low-resolution DETX/DETY background maps into high-resolution sky $(X / Y)$ images by interpolation. The user gives a template image containing the attitude information and the pixel numbers and sizes, and an associated event file (ideally the one used to create the template image file) for general header purposes. A rebinned background map is produced with the same resolution and at the same sky position as the template image. The radius of the circle over which interpolation is performed can be given (note that the background DETX/DETY maps are of 1' resolution, so an interpolation radius of this or slightly larger is recommended). An example of the task in use can be seen in Fig. 11.

\section{Event files}

As part of the analysis, for each observation, event files have been created, in the same manner as Lumb et al. (2002), filtered for times of high background and with all sources removed, as described in Sect. 3. The relevant event files have been 
$\mathbf{N}$

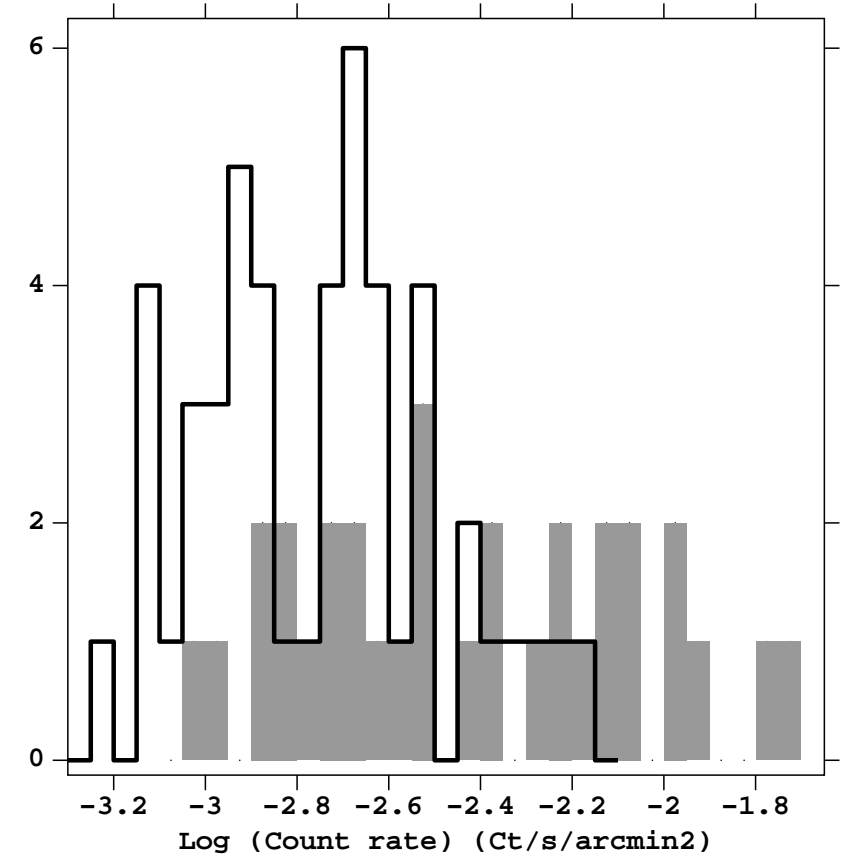

Fig. 10. (Thick line) the M1 0-band full-frame thin filter photon count rate distribution, and (filled region) the PN 0-band full-frameextended thin filter photon count rate distribution

merged together for each instrument/mode/filter combination (Tables 3 and 4), and eight separate event files have therefore been created (with nominal exposure times given as in Table 4), each having an extension containing a calibrated event list in the same format as produced by the XMM-SAS. The event files have had sky coordinates assigned to them for a pointing on the sky of RA $=0$, Dec $=0, P A=0$. These event files (or indeed any) can be reprojected onto any point in the sky via e.g. skycast (see http://www.sr.bham.ac.uk/xmm3/).

It is believed that these event files offer several improvements over previous versions (e.g. those of Lumb et al. 2002, themselves improvements on previously-created versions) for several reasons. Important points are as follows (note that many of these points apply not only to the event files, but also to the background maps discussed above);

- The event files have been created separately for each combination of instrument, instrument mode and filter. Hence, event files now exist for medium filter and also for pn full-frame-extended mode, neither of which had existed previously.

- As stated in the analysis sections, all data are collected from source-subtracted and high-background filtered fields with no bright sources or diffuse features, which could contaminate the "background", even after source-subtraction.

- The datasets are longer than previously available event files (over a million seconds of clean low-background data exists in each of the thin filter MOS datasets, for example). This improves signal-to-noise.

- The secondary extensions of the files have been removed, and all headers cleaned and corrected. This results in far smaller and more manageable files. It is possible to treat the event files as normal SAS event files; images, spectra and lightcurves can be created via evselect, and one can run ftools on the files.

- The event files combine a large number (see Table 4) of observations (rather than a few, long observations), such that the holes left by the source-subtraction are of less importance, and are heavily diluted by data from other observations. Due partly to the different exposure times of the individual observations, no single flux limit exists above which sources have been removed. However, flux histograms of the detected and thereafter removed sources do show quite a sharp cutoff at $\approx 1 \times 10^{-14} \mathrm{erg} \mathrm{cm}^{-2} \mathrm{~s}^{-1}$ (see Fig. 12).

- The exposure times are believed to be accurate, and incorporate the effects of losses due to deadtime. For each event file, 4 " resolution exposure maps (in DETX/DETY coordinates as described in Table 2) are also available from the web URL http://www.sr.bham.ac.uk/xmm3/. These are useful in evaluating the degree of the source and bad pixel removal, and calculating the exact value of the exposure of the background event files in a particular region of the detector. They are named in a similar manner to the event files (e.g. expmap_ft0000_M1.fits is a MOS1 fullframe, thin filter exposure map). The eight exposure maps are shown in Fig. 13.

\subsection{Event files: Usage and caveats}

The eight event files are available from http://www.sr.bham.ac.uk/xmm3/ and are named in a similar manner to the background maps. E1_ft0000_M1.fits is a MOS1 full-frame, thin filter event file. PN and M2 are for the pn and MOS2 instruments, an e denotes extended full-frame mode, and an m, medium filter.

Before using the event files for background analysis, it is strongly suggested consulting Lumb et al. (2002). Because of the above improvements, a number of the caveats noted by Lumb et al. do not apply to the present files. Many however, are still valid:

- Though the extraction of the background can be done in detector coordinates, the background event files can be skycast onto the sky position of the user's field (see http: //www.sr.bham.ac.uk/xmm3/ - alternatively use the XMMSAS task attcalc).

- Remnant low-level flares will remain in the event files. It is known, for instance, that at low energies, the proton flares turn on more slowly, but earlier than at higher energies. The user could apply further, more stringent flare-screening to the event files than that described in Sect. 3.2, but note that the lowest level proton fluxes may be spectrally variable, so that no complete subtraction may be possible.

- Although point sources have been removed, examination of images created from the datasets does reveal fluctuations in intensity. Over scales of arcminutes appropriate to extended sources, this is not expected to be a significant problem, and indeed representative of unresolved background. If a user however, should try to extract spectra from regions comparable to the PSF scale, care should be taken, and a 


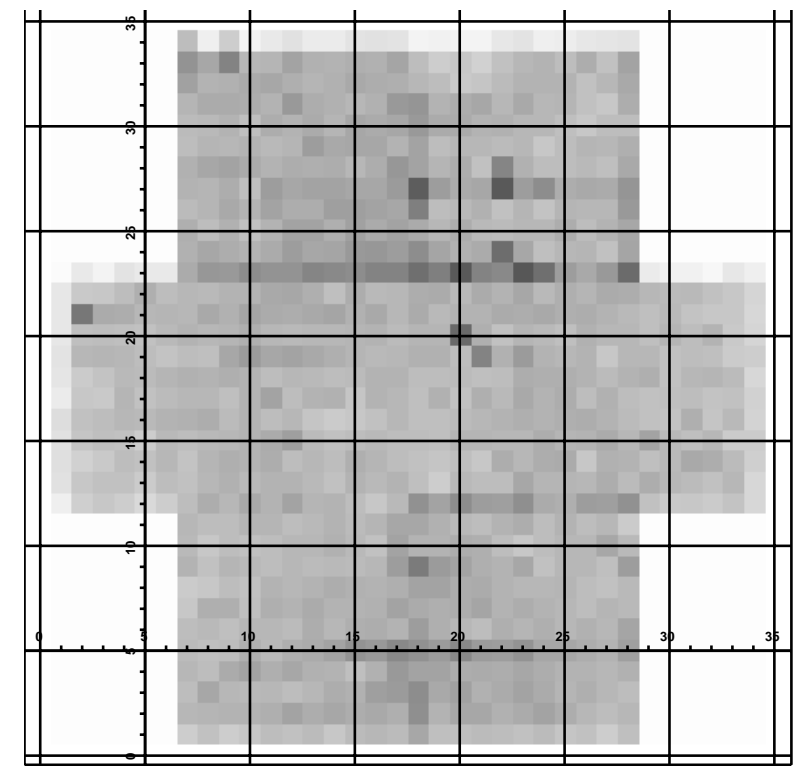

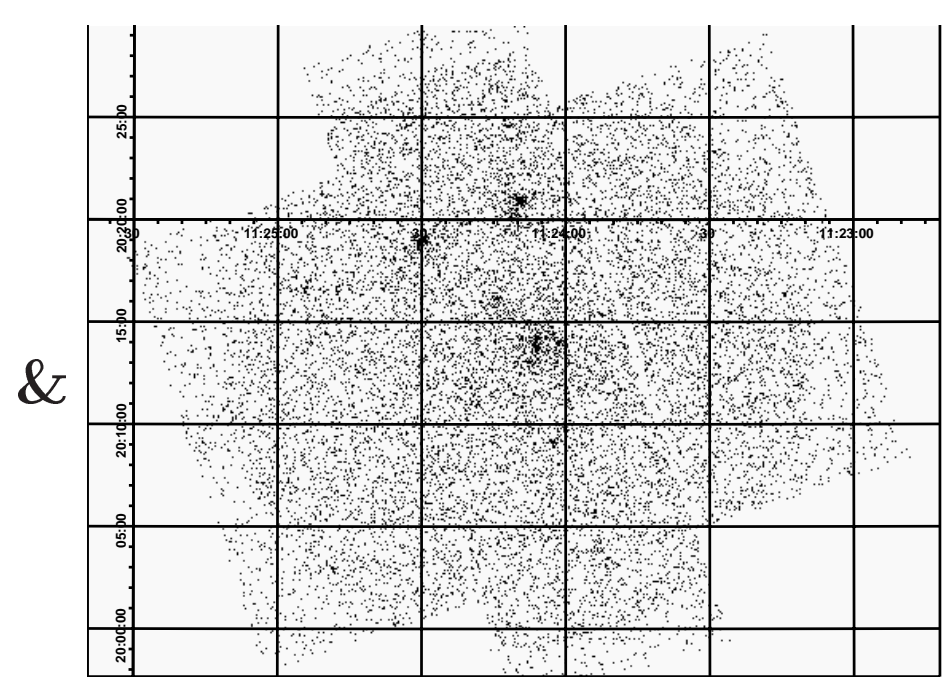

User's source image (Sky; ?")

BG image (DETX/Y; $\left.1^{\prime}\right)$

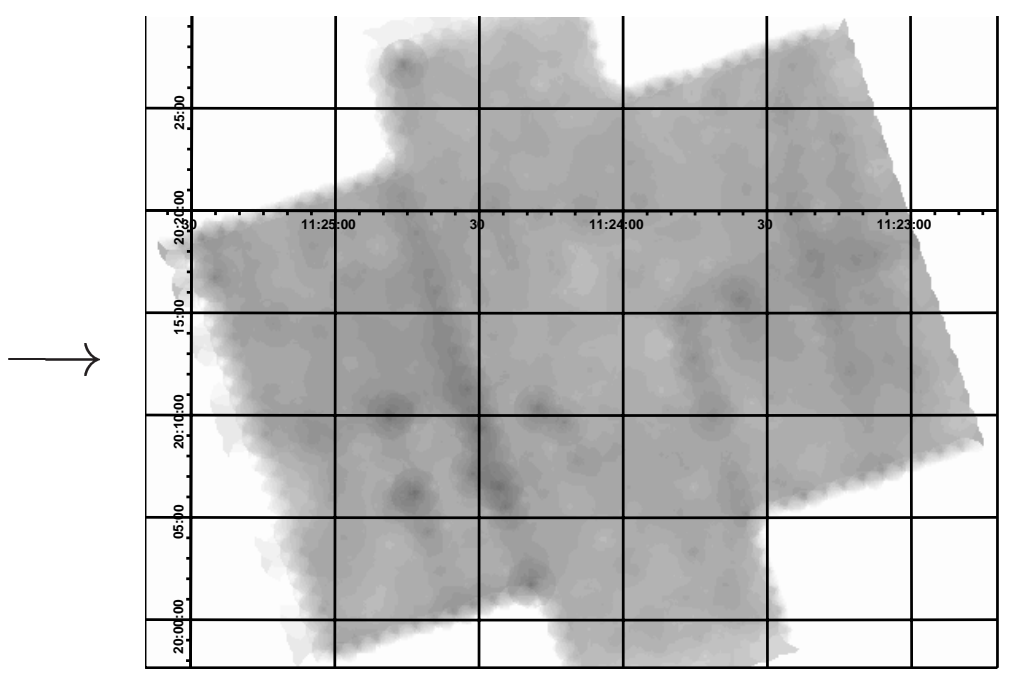

Rebinned \& sky-projected BG image (Sky; ?")

Fig. 11. Usage of BGrebinimage $2 S K Y$ to rebin and sky-project a given low-resolution DETX/Y background map (top left, specifically the M1 thin-filter 0-band particle background map from Fig. 6) to the spatial binning and sky position of a user-created high-resolution sky image (top right). The resultant high-resolution sky background image is shown at the bottom. The dark areas in the background map are due mainly to CCD gaps.

manual inspection may be necessary to guard against a local excess or deficit of counts arising from the point source extraction procedures. In some of the present cases, it is true that, as many of the observations were pointed such that the target source was at the centre of the detector, a deficit in counts (by as much as $50 \%$ or greater) is seen at this position. This can be corrected for by making use of the exposure maps provided (Fig. 13).

- Many defects are seen at the lowest energies (below $0.3 \mathrm{keV}$ ), and the calibration of the EPIC response at these energies is not so well understood as it is at higher energies. Care should be taken when performing analysis at $\therefore 0.25 \mathrm{keV}$.

\section{Future developments}

At the time of writing, there has been only limited experience gained in using the maps, the event files and the related software. Any feedback on using the datasets would be very useful and is most welcome (please email any comments to amr30@star.le.ac.uk).

It is hoped that further releases of the datasets, created using larger numbers of pointed observations, and perhaps using further modes and filters, will be made available in the future. This will be announced in the usual manner and via the URL http://www.sr.bham.ac.uk/xmm3/. 

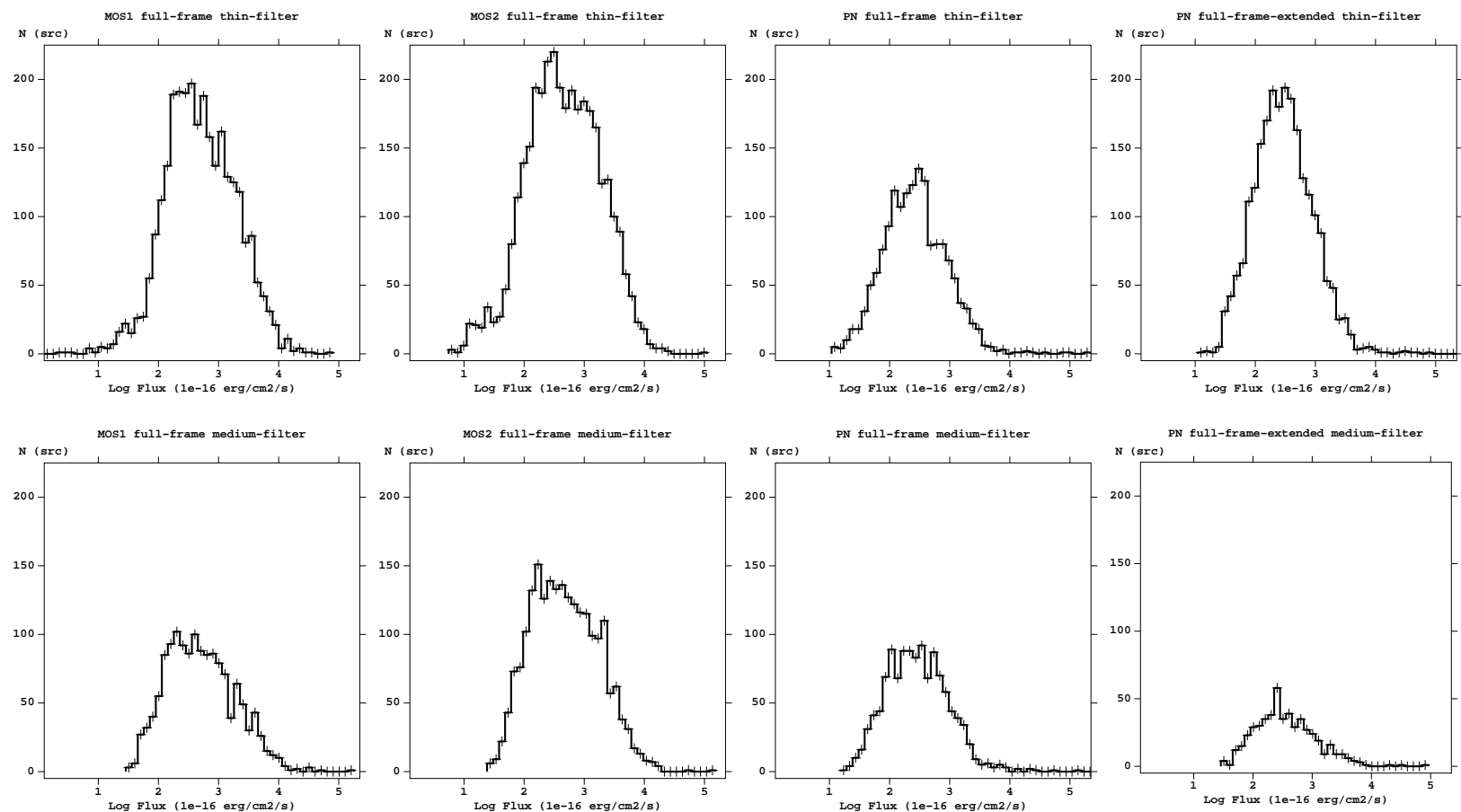

Fig. 12. Flux histograms of the detected and thereafter removed sources for each of the instrument/mode/filter combinations. (Top) Thin filter: MOS1, MOS2 and pn full-frame, pn full-frame extended. (Bottom) the same but for medium filter. All plots are to the same scale.
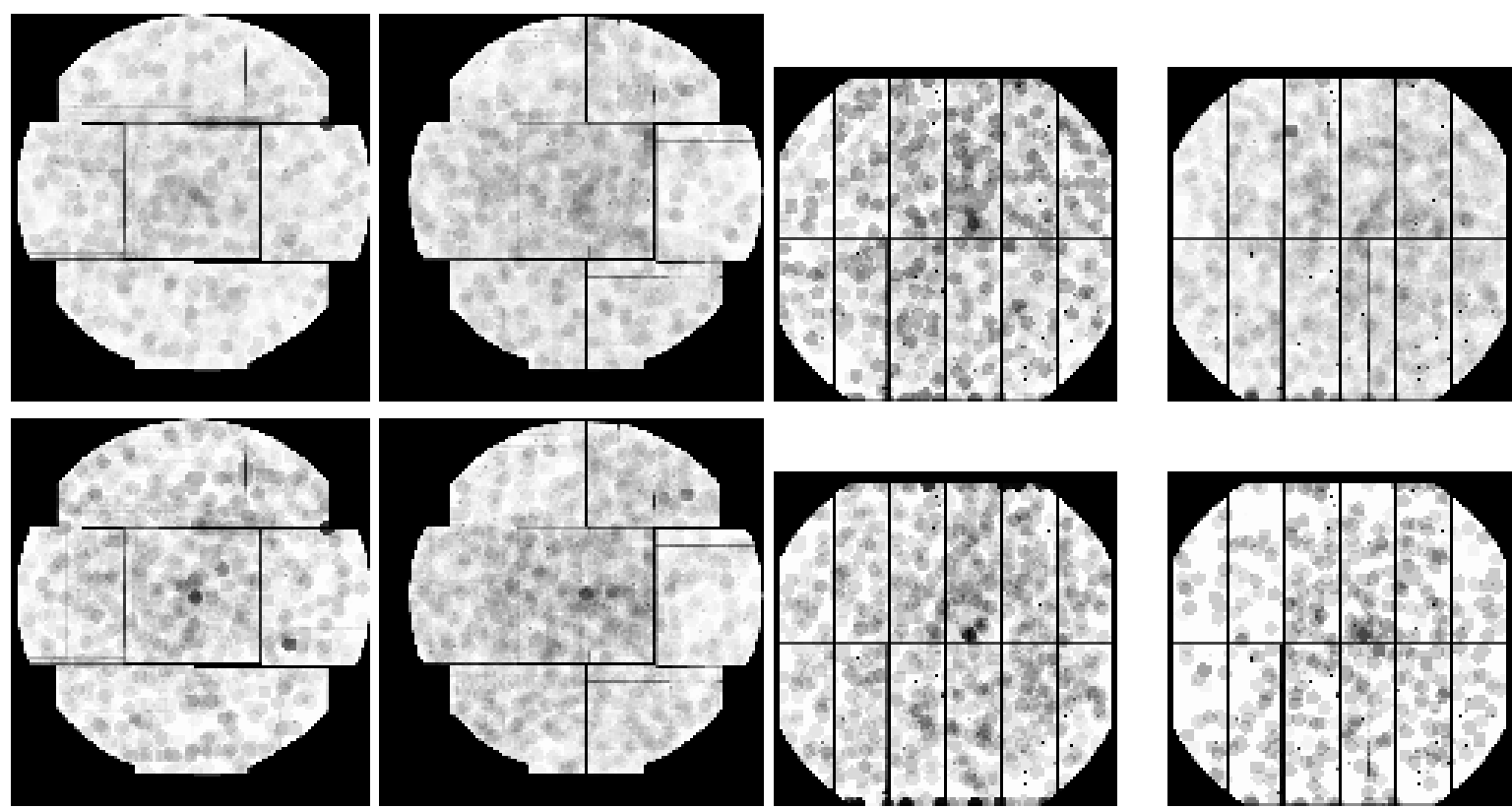

Fig. 13. 4" resolution DETX/DETY (see Table 2) exposure maps for the background event files in each of the instrument/mode/filter combinations. (Top) Thin filter: MOS1, MOS2 and pn full-frame, pn full-frame extended. (Bottom) the same but for medium filter.

Acknowledgements. This paper is partially based on data which are proprietary. These data were made available by the XMM-Newton project scientist specifically for this purpose. We wish to thank Monique Arnaud, Jean Ballet, Laurence Jones, Dave Lumb, Silvano Molendi, Wolfgang Pietsch \& Steve Snowden for very useful discussions during this work, and Mike Denby for help in obtaining many of the XMM datasets. We also thank the referee, Fred Jansen, for useful comments which have improved the paper. AMR acknowledges the support of PPARC funding.

\section{Appendix: The XMM-Newton EPIC X-ray back- ground: Additional notes}

There have been various studies of the XMM-Newton EPIC background, and many of these are described in Marty (2002), Lumb (2002) \& especially Lumb et al. (2002). 
A number of datasets have also been analysed and merged together, in a similar manner to that presented here. Two sets of data are particularly relevant to the present paper:

(i) Background datasets have been produced (Lumb 2002; Lumb et al. 2002) for the three EPIC instruments by source-subtracting and co-adding a few long observations. These event files can be obtained from http://xmm.vilspa.esa.es/ccf/epic/\#background .

(ii) Event lists combining several CLOSED observations have been created by Phillipe Marty, and these have proven very useful in the analysis and background-subtraction of extended objects (see Sect. 3.2). These data can be obtained from ftp: //www-station.ias.u-psud.fr/pub/epic/Closed

In addition, several novel methods have been used to analyse very extended and diffuse X-ray sources, where the extraction of the background is difficult. Many of these are described in Marty et al. 2002. Of special interest are the works of Arnaud et al. (2001, 2002), de Luca \& Molendi (2002), Ghizzardi et al. (2000), Molendi (2001), and Pratt \& Arnaud (2002).

\section{References}

Arnaud, M., Neumann, D. M., Aghanim, N., et al. 2001, A\&A, 365, L80

Arnaud, M., Majerowicz, S., Lumb, D., et al. 2002, A\&A, 390, 27

de Luca, A., \& Molendi, S. 2002, Proc. of the Symp. New Visions of the X-ray Universe in the XMM-Newton and Chandra era, [astro-ph/0202480]

Ghizzardi, S. 2000, EPIC-MCT-TN-003

Jansen, F., Lumb, D., Altieri, B., et al. 2001, A\&A, 365, L1

Lumb, D. 2002, http://xmm.vilspa.esa.es/docs/documents/ CAL-TN-0016-2-0.ps.gz

Lumb, D., Warwick, R. S., Page, M., \& de Luca, A. 2002, A\&A, 389, 93

Marty, P. B., Kneib, J. -P., Sadat, R., Ebeling, H., \& Smail, I. 2002, Proc. SPIE, 4851, 208

Molendi, S. 2001, http://xmm.vilspa.esa.es/sas/workshops/ estec2001/smolendi/smolendi-viewgraphs/slide.ps.gz Pratt, G., \& Arnaud, M. 2002, A\&A, 394, 375

Strüder, L., Briel, U., Dennerl, K., et al. 2001, A\&A, 365, L18

Turner, M. J. L., Abbey, A., Arnaud, M., et al. 2001, A\&A, 365, L27 